\begin{tabular}{|r|l|}
\hline \multicolumn{2}{|c|}{ Statistica Sinica Preprint No: SS-2021-0280 } \\
\hline Title & $\begin{array}{l}\text { Spatial-temporal Model with Heterogeneous Random } \\
\text { Effects }\end{array}$ \\
\hline Manuscript ID & SS-2021-0280 \\
\hline URL & http://www.stat.sinica.edu.tw/statistica/ \\
\hline DOI & $10.5705 /$ ss.202021.0280 \\
\hline Complete List of Authors & $\begin{array}{l}\text { Xingdong Feng, } \\
\text { Wenyu Li and } \\
\text { Qianqian Zhu }\end{array}$ \\
\hline Corresponding Author & Qianqian Zhu \\
\hline E-mail & zhu.qianqian@mail.shufe.edu.cn \\
\hline Notice: Accepted version subject to English editing. \\
\hline
\end{tabular}




\title{
Spatial-temporal Model with Heterogeneous Random Effects
}

\author{
Xingdong Feng, Wenyu Li and Qianqian Zhu \\ School of Statistics and Management \\ Shanghai University of Finance and Economics
}

\begin{abstract}
In this paper, we propose a novel spatial-temporal model with individual random effects characterized by a location-scale structure, which allows us to flexibly capture the pure influence of space-specific factors in the framework of quantile regression. A hybrid two-stage estimation procedure is introduced for this model, where the first stage proposes a Gaussian quasi-maximum likelihood estimator (QMLE) for the spatial-temporal effects while the second stage constructs a weighted conditional quantile estimator (WCQE) to study the conditional quantiles of the random effects related to space-specific attributes. The validity of the two-stage hybrid estimation is verified, and the asymptotic properties of our estimators are established. Our simulation study indicates that the proposed estimation procedure performs well in different scenarios with finite samples, and a real case study on the air quality of China is used to illustrate the application of the proposed method.
\end{abstract}

Keywords: Dynamic spatial autoregressive models; Hybrid estimation; Quantile regression; Quasimaximum likelihood estimation; Random effects.

\section{Introduction}

With the rapid development of world economy and gradual deterioration of global ecological environment, countries around the world have taken measures such as establishing and improving environmental protection mechanisms to maintain the ecological equilibrium (Streck, 2004; Busch and Jörgens, 2005). Meanwhile, urban air qualities have attacked in- 
tensive attentions (Wang and Hao, 2012; Gulia et al., 2015) since poor air qualities impose adverse impacts on human health and survival (Cakmak et al., 2011; Bowatte et al., 2015). Researchers have increasingly focused on the quantitative analysis of the impact of social and anthropogenic driving force on urban air qualities. For example, De Bruyn et al. (1998) and Chen and $\mathrm{Xu}$ (2017) studied the relationship between the air quality and the economic development; Cramer (1998) and Chen et al. (2020) investigated the influence of population density on the air quality; Li et al. (2018) and Yu and Liu (2020) considered the impact of industrial structure change on the air quality. However, as a complex and large system, the urban ambient air system is not only affected by human activities, but also closely related to spatial-temporal effects including historical patterns, geographical location, meteorological conditions and so on (Yang et al., 2007; Giri et al., 2008; Zhou et al., 2022). As a result, exploring the social driving force of urban air quality pressure after removing spatial-temporal effects becomes meaningful and necessary.

In literatures, spatial effects which depict the geographical correlation, are usually modeled in three forms: (i) spatial autoregressive (SAR) models with the spatial effect built at the response variable (Lee, 2004; Yu et al., 2008); (ii) spatial error models with the spatial effect modeled in the disturbance term (Kapoor et al., 2007; Kelejian and Prucha, 2010; Su and Yang, 2015); and (iii) spatial panel data models with the spatial effect simultaneously reflected in both responses and errors (Kelejian and Prucha, 1998, 1999). Some of previous models include temporal effects which capture autocorrelation in the form of lagged response by the same way as in time series models. As the practical need for analyzing spatial-temporal data increases, spatial panel data models including spatial and temporal effects simultaneously have been widely studied. For example, Yu et al. (2008) considered 
the Gaussian quasi-maximum likelihood estimator (QMLE) for the spatial dynamic panel data (SDPD) model with fixed effects, when both the individual dimension $N$ and time dimension $T$ are large; Lee and $\mathrm{Yu}(2014)$ proposed the generalized method of moments estimators for the SDPD model with fixed effects, when $N$ is large and $T$ can be large but small relative to $N$; and Su and Yang (2015) studied the QMLE for dynamic panel models with spatial errors and random/fixed effects when $N$ is large but $T$ is fixed. In this paper, we propose a dynamic spatial autoregressive (DSAR) model to capture the spatial-temporal effects and investigate its estimation.

For spatial panel data models, the individual effect is another important ingredient, which is widely investigated in form of fixed effect (Yu et al., 2008; Lee and Yu, 2010; Shi and Lee, 2017) or random effect (Baltagi et al., 2013; Su and Yang, 2015). Both fixed and random effects have merits and demerits: the random effect model avoids the "incidental parameter" problem (Neyman and Scott, 1948) in fixed effect models and has more efficient estimators, but it neglects possible correlations between individual effects and regressors; meanwhile, estimators of fixed effect models are robust in a sense that the fixed effect can be arbitrarily related to regressors (Mundlak (1978); Li and Yang (2021); Page 624 of Hansen (2021)). Note that the random effects in previous literatures are assumed to be with zero means and constant variances, which implies that the random effects are homogeneous for individuals. However, individual random effects can be influenced by space-specific covariates and this possibly makes the random effects heterogeneous. For example, the influence of socio-economic factors on air qualities possesses individual heterogeneity among cities. Generally heterogeneity across individuals can be described by fixed effects or heteroskedastic random effects (Baltagi et al., 2010). Both fixed and heteroskedastic random effects 
only emphasize the difference among individuals, but cannot help to explore information from the heterogeneity. Motivated by this fact, this paper alternatively considers a spacespecific-covariates-dependent location-scale structure for the individual effect. Specifically, the location of individual effect depicts the difference among individuals on the conditional mean and can be arbitrarily related to time-variant regressors, thus it can be viewed as a fixed effect; whereas the scale of individual effect is random and allows for conditional heteroscedasticity. Hence the location-scale structure combines advantages of fixed and random effects. Moreover, it can flexibly extract the influence of space-specific covariates on the whole distribution of the individual random effect.

It is well known that quantile regression (Koenker and Bassett, 1978) is a popular tool to capture heterogeneity and exploit the distributional information among data, which can provide a complete picture for studying influence of covariates on response. Reconsider the example of the air quality, we are interested in exploring the influence of socio-economic factors on the air quality after removing spatial-temporal effects, not only on the average level but also on the different quantile levels especially the lower and upper ones. This motivates us to adopt quantile regression for the proposed individual effects in location-scale structure. In summary, we propose a new framework to capture individual heterogeneity in the presence of spatial-temporal effects, and explore influence of space-specific covariates on response. The contributions of this paper are threefold.

(a) We propose a DSAR model with possibly heterogeneous random effects. To our best knowledge it is the first time that the random effect of spatial models is assumed to have a location-scale structure that can be affected by space-specific covariates. As a result, this model can capture the pure influence of some space-specific factors on 
different quantile levels of the response with spatial-temporal effects removed.

(b) We introduce a two-stage hybrid estimation procedure for the proposed model. Specifically, a QMLE is proposed in the first stage to estimate spatial-temporal effects, and a weighted conditional quantile regression estimator (WCQE) is designed in the second stage by replacing unobserved random effects with observed pseudo ones based on the QMLE.

(c) The theoretical contributions can be summarized as follows: (i) we derive the consistency and asymptotic normality for the QMLE; (ii) we establish the weak convergence of the quantile regression process and the asymptotic distribution of the WCQE; (iii) two estimators are proposed for the conditional scale coefficients of random effects and their asymptotic results are established.

The remainder of the paper is organized as follows. Section 2 introduces the model. Section 3 proposes a two-stage hybrid estimation procedure for this model, and establishes all related asymptotic results. A simulation study is conducted in Section 4 to assess the finite sample performance of the estimation procedure, and an empirical analysis on the air quality is provided in Section 5 to illustrate the usefulness of the proposed model and its inference tools. Conclusion and discussion are made in Section 6. A bootstrapping procedure for the QMLE as well as the technical details are relegated to the Supplementary Material. Throughout this paper, $\otimes$ denotes the Kronecker product of two matrices, $|\cdot|$ denotes the absolute value of a scalar/vector or the determinant of a matrix, $\|\cdot\|$ denotes the Euclidean norm of a vector, and $\operatorname{tr}(\cdot)$ denotes the trace of a matrix. Let $\lambda_{\min }(\cdot)$ and $\lambda_{\max }(\cdot)$ be the smallest and largest eigenvalues of a matrix, respectively. Denote $N$ and $T$ as the total numbers of spatial units and time periods, respectively. Define $I(\cdot)$ as the indicator function. 
For a positive integer $m$, denote $I_{m}$ as the $m \times m$ identity matrix, $\mathbf{0}_{m}$ as the $m \times 1$ vector of zeros, $\boldsymbol{\iota}_{m}$ as the $m \times 1$ vector of ones, and let $J_{m}=\boldsymbol{\iota}_{m} \boldsymbol{\iota}_{m}^{\prime}$. The operator $E$ denotes the expectation with respect to the probability measure, $\mathbb{E}_{n}$ denotes the expectation with respect to the empirical measure, and $\mathbb{G}_{n}=\sqrt{n}\left(\mathbb{E}_{n}-E\right) \cdot \ell^{\infty}(\mathcal{T})$ denotes the space of all uniformly bounded functions on $\mathcal{T}$. Moreover, $\rightarrow_{p}$ denotes the convergence in probability, $\rightarrow_{d}$ denotes the convergence in distribution, and $\rightsquigarrow$ denotes the weak convergence. In addition, an $\mathrm{R}$ package implementing the proposed two-stage hybrid estimation procedure is available at https://github.com/wyLI2020/QuantileDSAR, and the dataset analyzed in Section 5 is also included.

\section{Model Specification}

Consider the dynamic spatial autoregressive (DSAR) model with random effects below,

$$
y_{i t}=\theta_{i}+\alpha y_{i, t-1}+\lambda \sum_{j=1}^{N} w_{N, i j} y_{j t}+\sum_{\ell=1}^{q} \gamma_{\ell} z_{\ell i t}+\varepsilon_{i t}, i=1, \ldots, N, t=1, \ldots, T,
$$

where $\theta_{i}$ 's are the space-specific or individual random effects, the scalar parameters $\alpha$ and $\lambda$ characterize the dynamic effect and spatial autoregressive effect, respectively, $\gamma_{\ell}$ 's are the coefficients of time-varying exogenous variables $\left\{z_{\ell i t}\right\}, w_{N, i j}$ 's are specified constant spatial weights that generate the spatial dependence, and $\varepsilon_{i t}$ 's are independent and identically distributed (i.i.d.) across $i$ and $t$ with mean zero and finite variance $\sigma_{\varepsilon}^{2}$. To flexibly depict the possible influence of space-specific covariates on the individual random effect, this paper assumes a location-scale structure for $\theta_{i}$ 's, that is,

$$
\theta_{i}=\psi_{0}+\sum_{\ell=1}^{p} \psi_{\ell} x_{\ell i}+\left(\beta_{0}+\sum_{\ell=1}^{p} \beta_{\ell}\left|x_{\ell i}\right|\right) \eta_{i}
$$

where $\left\{x_{\ell i}\right\}$ are time-invariant constant regressors, $\psi_{\ell}$ 's and $\beta_{\ell}$ 's are the coefficients of $\left\{x_{\ell i}\right\}$ and $\left\{\left|x_{\ell i}\right|\right\}$, respectively, $\beta_{0}>0, \beta_{\ell} \geq 0$ for $1 \leq \ell \leq p$, and $\eta_{i}$ 's are i.i.d. disturbances with 
mean zero and finite variance $\sigma_{\eta}^{2}$; see also Koenker and Zhao (1994) and Zhao and Xiao (2014). Let $\mu_{i}=\psi_{0}+\sum_{\ell=1}^{p} \psi_{\ell} x_{\ell i}$ and $\vartheta_{i}=\left(\beta_{0}+\sum_{\ell=1}^{p} \beta_{\ell}\left|x_{\ell i}\right|\right) \eta_{i}$, it then follows that

$$
\theta_{i}=\mu_{i}+\vartheta_{i}
$$

The location-scale structure combines the advantages of fixed and random effects. Specifically, the location $\mu_{i}$ depicts the difference among individuals on the conditional mean and can be arbitrarily related to time-variant regressors $\left\{z_{\ell i t}\right\}$, while the scale effect $\vartheta_{i}$ allows for conditional heteroscedasticity and reflects the influence of $\left\{x_{\ell i}\right\}$ on the fluctuation. Note that if $\psi_{\ell}=\beta_{\ell}=0$ for all $\ell=1, \ldots, p$, then $\theta_{i}$ will reduce to the commonly used individual random effect. Moreover, if $\beta_{0}=\beta_{1}=\cdots=\beta_{p}=0$, then $\theta_{i}$ will be the individual fixed effect captured by space-specific covariates with $p+1$ unknown parameters, which can avoid the "incidental parameters" problem due to $N$ unknown parameters in commonly used individual fixed effect models.

Denote $\boldsymbol{Y}=\left(\boldsymbol{y}_{1}^{\prime}, \ldots, \boldsymbol{y}_{T}^{\prime}\right)^{\prime}$ and $\boldsymbol{Y}_{-1}=\left(\boldsymbol{y}_{0}^{\prime}, \ldots, \boldsymbol{y}_{T-1}^{\prime}\right)^{\prime}$ with $\boldsymbol{y}_{t}=\left(y_{1 t}, \ldots, y_{N t}\right)^{\prime}, Z=$ $\left(Z_{1}^{\prime}, \ldots, Z_{T}^{\prime}\right)^{\prime}$ with $Z_{t}=\left(\boldsymbol{z}_{1 t}, \ldots, \boldsymbol{z}_{N t}\right)^{\prime}$ and $\boldsymbol{z}_{i t}=\left(z_{1 i t}, \ldots, z_{q i t}\right)^{\prime}, X=\left(\boldsymbol{x}_{1}, \ldots, \boldsymbol{x}_{N}\right)^{\prime}$ with $\boldsymbol{x}_{i}=$ $\left(1, x_{1 i}, \ldots, x_{p i}\right)^{\prime}$, and $\varepsilon=\left(\varepsilon_{1}^{\prime}, \ldots, \varepsilon_{T}^{\prime}\right)^{\prime}$ with $\varepsilon_{t}=\left(\varepsilon_{1 t}, \ldots, \varepsilon_{N t}\right)^{\prime}$. Let $S_{N T}(\lambda)=I_{T} \otimes B_{N}(\lambda)$ with $B_{N}(\lambda)=I_{N}-\lambda W_{N}$ and $W_{N}=\left\{w_{N, i j}, 1 \leq i, j \leq N\right\}$ being the $N \times N$ specified constant spatial weights matrix, and denote $\widetilde{Z}=\left(\boldsymbol{Y}_{-1}, Z,\left(\iota_{T} \otimes I_{N}\right) X\right)$. Then model (2.1) with (2.2) can be rewritten in the following matrix form:

$$
S_{N T}(\lambda) \boldsymbol{Y}=\widetilde{Z} \boldsymbol{\phi}+\left(\boldsymbol{\iota}_{T} \otimes I_{N}\right) \boldsymbol{\vartheta}+\boldsymbol{\varepsilon}
$$

where $\boldsymbol{\phi}=\left(\alpha, \boldsymbol{\gamma}^{\prime}, \boldsymbol{\psi}^{\prime}\right)^{\prime}$ with $\boldsymbol{\gamma}=\left(\gamma_{1}, \ldots, \gamma_{q}\right)^{\prime}$ and $\boldsymbol{\psi}=\left(\psi_{0}, \psi_{1}, \ldots, \psi_{p}\right)^{\prime}$, and $\boldsymbol{\vartheta}=\left(\vartheta_{1}, \ldots, \vartheta_{N}\right)^{\prime}$ with $\vartheta_{i}=\boldsymbol{x}_{a i}^{\prime} \boldsymbol{\beta} \eta_{i}, \boldsymbol{x}_{a i}=\left(1,\left|x_{1 i}\right|, \ldots,\left|x_{p i}\right|\right)^{\prime}$ and $\boldsymbol{\beta}=\left(\beta_{0}, \beta_{1}, \ldots, \beta_{p}\right)^{\prime}$. The following proposition provides a sufficient condition for the covariance stationarity of $\boldsymbol{y}_{t}$. 
Proposition 1. Suppose that $\eta_{i}$ and $\varepsilon_{i t}$ are mutually independent. If $\left|\alpha B_{N}^{-1}(\lambda)\right|<1$ and the process $\left\{\boldsymbol{z}_{i t}\right\}$ is covariance stationary, then there exists a unique covariance stationary solution to models (2.1) and (2.2). The solution is in form of

$$
\boldsymbol{y}_{t}=\sum_{m=0}^{\infty} \alpha^{m}\left[B_{N}^{-1}(\lambda)\right]^{m+1}\left(\boldsymbol{\theta}+Z_{t-m} \boldsymbol{\gamma}+\boldsymbol{\varepsilon}_{t-m}\right)
$$

Proposition 1 indicates that the stationarity of $\left\{\boldsymbol{y}_{t}\right\}$ restricts the spatial and temporal effects to some extent by imposing conditions on the weights matrix $W_{N}$, spatial effect parameter $\lambda$ and autoregressive coefficient $\alpha$. Specifically, $|\alpha|+|\lambda|<1$ implies $\left|\alpha B_{N}^{-1}(\lambda)\right|<1$ if $W_{N}$ is row-normalized. Moreover, the stationarity of $\left\{\boldsymbol{y}_{t}\right\}$ also depends on that of exogenous covariates $\left\{\boldsymbol{z}_{i t}\right\}$. But assuming both $\left\{\boldsymbol{y}_{t}\right\}$ and $\left\{\boldsymbol{z}_{i t}\right\}$ stationary makes the application of the proposed model very limited. This motivates us to consider an estimation method that is theoretically valid without any stationarity assumption on $\left\{\boldsymbol{y}_{t}\right\}$.

We are interested in the parameter estimation for $\boldsymbol{\phi}, \boldsymbol{\beta}, \lambda, \sigma_{\varepsilon}^{2}$ and $\sigma_{\eta}^{2}$, and more importantly in the conditional quantile estimation of individual random effects $\theta_{i}$ 's. Let $Q_{\eta}(\tau)$ be the $\tau$-th quantile of $\eta_{i}$. Denote $\boldsymbol{\theta}=\left(\theta_{1}, \ldots, \theta_{N}\right)^{\prime}, X_{a}=\left(\boldsymbol{x}_{a 1}, \ldots, \boldsymbol{x}_{a N}\right)^{\prime}$. The conditional quantile of $\boldsymbol{\theta}$, given the space-specific covariates $X$, has the form of

$$
Q_{\boldsymbol{\theta}}(\tau \mid X)=X \boldsymbol{\psi}+X_{a} \boldsymbol{\varphi}(\tau)
$$

where $\boldsymbol{\varphi}(\tau)=\left(\varphi_{0}(\tau), \varphi_{1}(\tau), \ldots, \varphi_{p}(\tau)\right)^{\prime}=Q_{\eta}(\tau) \boldsymbol{\beta}$. Notice that the individual effects $\boldsymbol{\theta}$ are unobservable, to estimate $Q_{\boldsymbol{\theta}}(\tau \mid X)$ in the framework of model (2.3), we propose a two-stage hybrid estimation procedure in the following section.

\section{Two-stage Hybrid Estimation}

The space-specific random effect $\theta_{i}$ is latent, so it is natural for the estimation under model (2.4) to obtain its observable approximate by (2.1) based on reasonable estimates of $\lambda, \alpha$ 
and $\boldsymbol{\gamma}$, and then apply the quantile regression estimation method to model (2.4) with $\theta_{i}$ replaced by its observable approximate to obtain estimates of $\boldsymbol{\psi}$ and $\varphi(\tau)$. Note that $\boldsymbol{\psi}$ is independent of $\tau$ and it is not necessary to estimate it by conditional quantile estimation in our model setting, while $\varphi(\tau)$ is $\tau$-dependent and can be estimated by the quantile estimation method. Moreover, $\lambda$ and $\phi$ can be estimated directly and thus the observable approximate of $\vartheta_{i}$ is easily obtained by model (2.3). These together with the fact that $Q_{\boldsymbol{\theta}}(\tau \mid X)=$ $X \boldsymbol{\psi}+Q_{\boldsymbol{\vartheta}}(\tau \mid X)$, motivate us to alternatively obtain observable approximates of $\vartheta_{i}$ 's and then apply quantile regression to them as given by

$$
Q_{\vartheta}(\tau \mid X)=X_{a} \varphi(\tau)
$$

Specifically, this paper adopts the QMLE for model (2.3), denoted by $\widehat{\boldsymbol{\zeta}}=\left(\widehat{\boldsymbol{\phi}}^{\prime}, \widehat{\sigma}_{\varepsilon}^{2}, \widehat{\lambda}, \widehat{\boldsymbol{\beta}}^{\star \prime}\right)^{\prime}$ for $\boldsymbol{\zeta}=\left(\boldsymbol{\phi}^{\prime}, \sigma_{\varepsilon}^{2}, \lambda, \boldsymbol{\beta}^{\star \prime}\right)^{\prime}$ with $\boldsymbol{\beta}^{\star}=\boldsymbol{\beta} \sigma_{\eta} / \sigma_{\varepsilon}$. Then the observable approximates of $\vartheta_{i}$ 's called the pseudo scale effects, denoted by $\widehat{\vartheta}_{i}$ 's, can be constructed based on $\widehat{\lambda}$ and $\widehat{\phi}$; see (3.11). We next build a quantile regression model for $\widehat{\vartheta}=\left(\widehat{\vartheta}_{1}, \ldots, \widehat{\vartheta}_{N}\right)^{\prime}$ using $X_{a}$ as the regressor, and construct a WCQE $\widehat{\varphi}(\tau)$ for $\boldsymbol{\varphi}(\tau)$ and a weighted quantile average estimator (WQAE) $\widehat{\boldsymbol{\beta}}$ for $\boldsymbol{\beta}$. In summary, a two-stage hybrid estimation procedure is proposed as follows:

Stage 1: Obtain the Gaussian QMLE $\widehat{\boldsymbol{\zeta}}$ based on model (2.3).

Stage 2: Define the pseudo scale effects $\left\{\widehat{\vartheta}_{i}\right\}$ by $(3.11)$ based on $\widehat{\boldsymbol{\zeta}}$. Then obtain the WCQE $\widehat{\boldsymbol{\varphi}}(\tau)$ for $\boldsymbol{\varphi}(\tau)$ based on model (3.1) with $\boldsymbol{\vartheta}$ replaced by $\widehat{\boldsymbol{\vartheta}}$, and propose the WQAE $\widehat{\boldsymbol{\beta}}$ for $\boldsymbol{\beta}$ by combing the information among $\widehat{\varphi}(\cdot)$ at multiple quantile levels $\tau$ 's.

As a result, if $S_{N T}(\widehat{\lambda})$ is invertible, then the conditional mean of the data $\boldsymbol{Y}$ can be estimated by $\widehat{E}(\boldsymbol{Y} \mid X)=S_{N T}^{-1}(\widehat{\lambda}) \widetilde{Z} \widehat{\boldsymbol{\phi}}$ based on model (2.3) and $\widehat{\boldsymbol{\zeta}}$. Moreover, the conditional quantile of random effects $\boldsymbol{\theta}$ can be estimated by $\widehat{Q}_{\boldsymbol{\theta}}(\tau \mid X)=X \widehat{\boldsymbol{\psi}}+X_{a}^{\prime} \widehat{\boldsymbol{\varphi}}(\tau)$ based on model (2.4), $\widehat{\boldsymbol{\psi}}$ 
and $\widehat{\varphi}(\tau)$. Statistical inference tools, such as significance tests for $\boldsymbol{\phi}, \boldsymbol{\varphi}(\tau)$ and $\boldsymbol{\beta}$ can also be constructed based on $\widehat{\boldsymbol{\phi}}, \widehat{\boldsymbol{\varphi}}(\tau)$ and $\widehat{\boldsymbol{\beta}}$. More details of the proposed estimation procedure and its asymptotics are refered to Sections 3.1 and 3.2 .

To establish the asymptotics for the proposed two-stage hybrid estimation procedure, the regularity conditions for $N$ and $T$ are assumed below.

Assumption 3.1. $T=c N^{r}$ for some constants $r \geq 0$ and $c>0$.

Assumption 3.2. $T=c N^{r}$ for some constants $0 \leq r \leq 1$ and $c>0$.

Assumption 3.3. $T=c N^{r}$ for some constants $0<r \leq 1$ and $c>0$.

Assumptions 3.1 and 3.2 allow $T$ to be fixed or goes to infinity as $N$ goes to infinity, and Assumption 3.2 restricts $T$ at a rate not greater than $N$ when it goes to infinity. Assumption 3.3 requires both $T$ and $N$ go to infinity, and it is a special case of Assumption 3.2. Specifically, the consistency of QMLE in the first stage is established under Assumption 3.1, while the asymptotic normality of QMLE is obtained under Assumption 3.2. For the asymptotic normality of QMLE, restricting the rate of $T$ (i.e. $T / N \rightarrow c^{\prime}$ for some constant $c^{\prime} \geq 0$ ) is needed to prove the asymptotic normality of $(N T)^{-1 / 2} \partial \ln \mathcal{L}_{N T}\left(\boldsymbol{\zeta}_{0}\right) / \partial \boldsymbol{\zeta}$ using Theorem A.1 of Kelejian and Prucha (2001); see details in the proof of Theorem 2 in the Supplementary Material. Moreover, since $T \rightarrow \infty$ is necessary for the validity of replacing $\boldsymbol{\vartheta}$ with $\widehat{\boldsymbol{\vartheta}}$ in quantile estimation, Assumption 3.3 is used to establish asymptotic results of WCQE and WQAE in the second stage; see details in Section 3.2.

\subsection{The Quasi-maximum Likelihood Estimation}

Recall that the parameter vector of model (2.3) is $\boldsymbol{\zeta}=\left(\boldsymbol{\phi}^{\prime}, \sigma_{\varepsilon}^{2}, \lambda, \boldsymbol{\beta}^{\star \prime}\right)^{\prime}$ with $\boldsymbol{\beta}^{\star}=\boldsymbol{\beta} \sigma_{\eta} / \sigma_{\varepsilon}$, and let $\boldsymbol{\zeta}_{0}=\left(\boldsymbol{\phi}_{0}^{\prime}, \sigma_{\varepsilon 0}^{2}, \lambda_{0}, \boldsymbol{\beta}_{0}^{\star \prime}\right)^{\prime}$ be the true value of $\boldsymbol{\zeta}$. Denote $\boldsymbol{V}_{N T}(\lambda, \boldsymbol{\phi})=S_{N T}(\lambda) \boldsymbol{Y}-$ 
$\widetilde{Z} \boldsymbol{\phi}, \boldsymbol{V}_{N T}=\left(\iota_{T} \otimes I_{N}\right) \boldsymbol{\vartheta}+\boldsymbol{\varepsilon}, B_{0 N}=B_{N}\left(\lambda_{0}\right)$ and $S_{0 N T}=S_{N T}\left(\lambda_{0}\right)$, then it follows that $\boldsymbol{V}_{N T}\left(\lambda_{0}, \boldsymbol{\phi}_{0}\right)=\boldsymbol{V}_{N T}=S_{0 N T} \boldsymbol{Y}-\widetilde{Z} \phi_{0}$. This paper considers the case that initial observations are generated exogenously, that is, $\boldsymbol{y}_{0}$ is exogenous such that it can be treated as a constant vector; see also Su and Yang (2015).

Assume that $\varepsilon_{i t}$ and $\eta_{i}$ are distributed with mean zeros and variances $\sigma_{\varepsilon}^{2}$ and $\sigma_{\eta}^{2}$, respectively, which implies that $\operatorname{cov}\left\{\boldsymbol{V}_{N T}(\lambda, \boldsymbol{\phi})\right\}=\sigma_{\varepsilon}^{2} \Omega_{N T}\left(\boldsymbol{\beta}^{\star}\right)$, where $\Omega_{N T}\left(\boldsymbol{\beta}^{\star}\right)=J_{T} \otimes A_{N}\left(\boldsymbol{\beta}^{\star}\right)+$ $I_{N T}$ with $A_{N}\left(\boldsymbol{\beta}^{\star}\right)=\operatorname{diag}\left\{\left(\boldsymbol{x}_{a 1}^{\prime} \boldsymbol{\beta}^{\star}\right)^{2}, \ldots,\left(\boldsymbol{x}_{a N}^{\prime} \boldsymbol{\beta}^{\star}\right)^{2}\right\}$. The Guassian quasi-likelihood function of $\zeta$ is then given by

$$
\mathcal{L}_{N T}(\boldsymbol{\zeta})=\left(2 \pi \sigma_{\varepsilon}^{2}\right)^{-\frac{N T}{2}}\left|S_{N T}^{\prime}(\lambda) \Omega_{N T}^{-1}\left(\boldsymbol{\beta}^{\star}\right) S_{N T}(\lambda)\right|^{\frac{1}{2}} \exp \left\{-\frac{\boldsymbol{V}_{N T}^{\prime}(\lambda, \boldsymbol{\phi}) \Omega_{N T}^{-1}\left(\boldsymbol{\beta}^{\star}\right) \boldsymbol{V}_{N T}(\lambda, \boldsymbol{\phi})}{2 \sigma_{\varepsilon}^{2}}\right\},
$$

and the corresponding quasi-log-likelihood function of $\zeta$ is

$$
\begin{aligned}
\ln \mathcal{L}_{N T}(\boldsymbol{\zeta})= & -\frac{N T}{2} \ln (2 \pi)-\frac{N T}{2} \ln \sigma_{\varepsilon}^{2}-\frac{1}{2} \ln \left|\Omega_{N T}\left(\boldsymbol{\beta}^{\star}\right)\right|+\ln \left|S_{N T}(\lambda)\right| \\
& -\frac{1}{2 \sigma_{\varepsilon}^{2}} \boldsymbol{V}_{N T}^{\prime}(\lambda, \boldsymbol{\phi}) \Omega_{N T}^{-1}\left(\boldsymbol{\beta}^{\star}\right) \boldsymbol{V}_{N T}(\lambda, \boldsymbol{\phi}) .
\end{aligned}
$$

Maximizing (3.2) gives the Gaussian QMLE of $\boldsymbol{\zeta}$, denoted by $\widehat{\boldsymbol{\zeta}}$. If $\varepsilon_{i t}$ and $\eta_{i}$ are normally distributed, then $\widehat{\zeta}$ is the MLE.

For easy computation, we consider the concentrated log-likelihood by concentrating out the $\boldsymbol{\phi}$ and $\sigma_{\varepsilon}^{2}$. Given $\boldsymbol{\delta}=\left(\lambda, \boldsymbol{\beta}^{\star \prime}\right)^{\prime}$, maximizing (3.2) leads to the following QMLEs of $\boldsymbol{\phi}$ and $\sigma_{\varepsilon}^{2}$

$$
\widehat{\boldsymbol{\phi}}(\boldsymbol{\delta})=\left(\widetilde{Z}^{\prime} \Omega_{N T}^{-1}\left(\boldsymbol{\beta}^{\star}\right) \widetilde{Z}\right)^{-1} \widetilde{Z}^{\prime} \Omega_{N T}^{-1}\left(\boldsymbol{\beta}^{\star}\right) S_{N T}(\lambda) \boldsymbol{Y}
$$

and

$$
\widehat{\sigma}_{\varepsilon}^{2}(\boldsymbol{\delta})=\frac{1}{N T} \widehat{\boldsymbol{V}}_{N T}^{\prime}(\boldsymbol{\delta}) \Omega_{N T}^{-1}\left(\boldsymbol{\beta}^{\star}\right) \widehat{\boldsymbol{V}}_{N T}(\boldsymbol{\delta}),
$$

where $\widehat{\boldsymbol{V}}_{N T}(\boldsymbol{\delta})=S_{N T}(\lambda) \boldsymbol{Y}-\widetilde{Z} \widehat{\boldsymbol{\phi}}(\boldsymbol{\delta})$. Then by plugging (3.3) and (3.4) in (3.2), the concen- 
trated log-likelihood function of $\boldsymbol{\delta}$ is

$$
\ln \ell_{N T}(\boldsymbol{\delta})=-\frac{N T}{2}(\ln (2 \pi)+1)-\frac{N T}{2} \ln \widehat{\sigma}_{\varepsilon}^{2}(\boldsymbol{\delta})-\frac{1}{2} \ln \left|\Omega_{N T}\left(\boldsymbol{\beta}^{\star}\right)\right|+\ln \left|S_{N T}(\lambda)\right| .
$$

The QMLE $\widehat{\boldsymbol{\delta}}$ which maximizes the function (3.5) is given by

$$
\widehat{\boldsymbol{\delta}}=\arg \max _{\boldsymbol{\delta} \in \Delta} \ln \ell_{N T}(\boldsymbol{\delta})
$$

where $\Delta$ is the parameter space of $\boldsymbol{\delta}$, which is assumed to be compact and the true value $\boldsymbol{\delta}_{0}=\left(\lambda_{0}, \boldsymbol{\beta}_{0}^{\star \prime}\right)^{\prime}$ is in the interior of $\Delta$. Then the QMLEs of $\boldsymbol{\phi}$ and $\sigma_{\varepsilon}^{2}$ are $\widehat{\boldsymbol{\phi}}=\widehat{\boldsymbol{\phi}}(\widehat{\boldsymbol{\delta}})$ and $\widehat{\sigma}_{\varepsilon}^{2}=$ $\widehat{\sigma}_{\varepsilon}^{2}(\widehat{\boldsymbol{\delta}})$, respectively. As a result, the QMLE of $\boldsymbol{\zeta}$ is given by $\widehat{\boldsymbol{\zeta}}=\left(\widehat{\boldsymbol{\phi}}^{\prime}, \widehat{\sigma}_{\varepsilon}^{2}, \widehat{\boldsymbol{\delta}}^{\prime}\right)^{\prime}=\left(\widehat{\boldsymbol{\phi}}^{\prime}, \widehat{\sigma}_{\varepsilon}^{2}, \widehat{\lambda}, \widehat{\boldsymbol{\beta}}^{\star \prime}\right)^{\prime}$.

To establish the consistency and asymptotic normality for the QMLE, we introduce the following assumptions.

Assumption 3.4. (i) $E\left|\varepsilon_{i t}\right|^{4+\epsilon_{0}}<\infty$ and $E\left|\eta_{i}\right|^{4+\epsilon_{0}}<\infty$ for some $\epsilon_{0}>0$; (ii) $\varepsilon_{i t}$ and $\eta_{i}$ are mutually independent.

Assumption 3.5. (i) The elements $w_{N, i j}$ of $W_{N}$ are bounded constants uniformly for all $N$ in all $i$ and $j$. As a normalization, $w_{N, i i}=0$ for all $i$; (ii) The matrix $B_{0 N}$ is nonsingular; (iii) The sequences of matrices $\left\{W_{N}\right\}$ and $\left\{B_{0 N}^{-1}\right\}$ are bounded in both row and column sums uniformly for all $N$; (iv) $\left\{B_{N}^{-1}(\lambda)\right\}$ are bounded in either row or column sums uniformly for all $N$, uniformly in $\lambda \in \Delta(\lambda)$; (v) The sequence of matrices $\left\{\sum_{t=1}^{T} \alpha_{0}^{t-1}\left(B_{0 N}^{-1}\right)^{t}\right\}$ are uniformly bounded for all $N$ and $T$ in both row and column sums.

Assumption 3.6. (i) $x_{\ell i}$ 's are uniformly bounded constants for all $N$; (ii) $E\left(z_{\ell i t}\right)=0$ and $E\left|z_{\ell i t}\right|^{4+\epsilon_{0}}<\infty$ for some $\epsilon_{0}>0$; (iii) For any $1 \leq \ell \leq q$, the covariance matrix of $\left\{\left|z_{\ell i t}\right|, 1 \leq i \leq N, 1 \leq t \leq T\right\}$ is uniformly bounded in row sums, and it holds that

$$
\sum_{1 \leq i_{3}, i_{4} \leq N} \sum_{1 \leq t_{3}, t_{4} \leq T} \operatorname{cov}\left(z_{\ell i_{1} t_{1}} z_{\ell i_{2} t_{2}}, z_{\ell i_{3} t_{3}} z_{\ell i_{4} t_{4}}\right)=O(1)
$$


for any $1 \leq i_{1}, i_{2} \leq N$ and $1 \leq t_{1}, t_{2} \leq T$; (iv) $\frac{1}{N T} \widetilde{Z} \widetilde{Z}^{\prime}$ is positive definite for sufficiently large $N$ and $\lambda_{\min }\left(\frac{1}{N T} \widetilde{Z}^{\prime} \Omega_{N T}^{-1}\left(\boldsymbol{\beta}^{\star}\right) \widetilde{Z}\right)$ is uniformly bounded away from zero almost surely (a.s.).

Assumption 3.4 provides basic conditions on the disturbances, which are standard in the random effects panel data literature; see Assumptions $\mathrm{R}(i)$ and $\mathrm{R}(\mathrm{ii})$ of $\mathrm{Su}$ and Yang (2015). Note that Assumption 3.4 $(i)$ does not require $\varepsilon_{i t}$ 's to be identically distributed. Since the heterogeneity among individuals is captured by the random effects $\theta_{i}$ 's in model (2.1), we assume $\varepsilon_{i t}$ 's have a common variance instead of $i$-dependent variances.

Assumption 3.5 provides the essential features of the weights matrix which are commonly assumed for spatial models; see also Assumption G2 of Su and Yang (2015) and Assumptions 2-5 of Lee (2004). In empirical applications, $W_{N}$ is usually row normalized in such that $B_{N}(\lambda)$ is nonsigular for $\lambda \in(-1,1)$; see also Kelejian and Prucha (2010). Invertibility of $B_{0 N}$ in Assumption 3.5 $(\mathrm{ii})$ guarantees that (2.3) has an equilibrium and the disturbance term is well defined. Assumption 3.5( facilitates the study of the asymptotic properties of the spatial parameter estimators, and Assumption 3.5 $(i v)$ is required to establish the consistency. Assumption 3.5 $(v)$ is implied by Assumption $3.5($ iii $)$ when $T$ is fixed. When both $N$ and $T$ go to infinity, Assumption 3.5 (v) is necessary to guarantee that the variances of $y_{i t}$ 's are bounded, and it is an extension of Assumption 3.5 (iii) which guarantees that the variances of $y_{i}^{\prime}$ 's in the SAR model are bounded as $N$ goes to infinity (Lee, 2004). Note that Assumption 3.5 (iii) implies that both row and column sums of $\left(B_{0 N}^{-1}\right)^{t}$ are bounded for an arbitrary $t$. This together with $\left|\alpha_{0}\right|<1$, implies that Assumption 3.5 $(v)$ holds as long as $\alpha_{0}^{t-1}$ decreases faster than the row or column sum of $\left(B_{0 N}^{-1}\right)^{t}$ increases as $t$ increases, which is usually satisfied in practice. Moreover, if $W_{N}$ is a row-normalized matrix, then both $\left\{B_{N}^{-1}(\lambda)\right\}$ and $\left\{\sum_{t=1}^{T} \alpha^{t-1}\left[B_{N}^{-1}(\lambda)\right]^{t}\right\}$ are uniformly 
bounded in row sums for $|\alpha|+|\lambda|<1$; see also Lee (2004). In addition, Assumption 3.5 actually implies some restrictions on the process $\left\{\boldsymbol{y}_{t}\right\}$ by imposing conditions on the weights matrix $W_{N}$, spatial effect parameter $\lambda$ and autoregressive coefficient $\alpha$. But both stationary and nonstationary cases of $\left\{\boldsymbol{y}_{t}\right\}$ are allowable under Assumption 3.5.

The conditions on regressors in Assumptions 3.6 (i), (ii) and (iv) are general and parallel Assumption 6 in Lee (2004), Assumption 4 in Yu et al. (2008) and Assumptions G1(iii) and $\mathrm{R}\left(i v^{\star}\right)$ in Su and Yang (2015), where regressors in Lee (2004) are time-invariant constants, in Yu et al. (2008) are time-variant constants, and in Su and Yang (2015) are time-invariant or time-variant random variables. Note that Theorems 1 and 2 still hold if $x_{\ell i}{ }^{\prime}$ s $\left(z_{\ell i t}\right.$ 's $)$ are assumed either constants or random variables. Here we assume time-variant random $z_{\ell i t}$ 's but time-invariant constant $x_{\ell i}$ 's to emphasize the randomness by time $t$, and this also allows $x_{\ell i}$ to be arbitrarily related to $z_{\ell i t}$. Assumption 3.6(iii) is to guarantee that variances associated with $\left\{z_{\ell i t}, 1 \leq i \leq N, 1 \leq t \leq T\right\}$ are $O(N T)$; see details in Lemmas 1 - 6. Particularly, (3.6) implies that given $\left(i_{1}, t_{1}\right)$ and $\left(i_{2}, t_{2}\right), z_{\ell i_{1} t_{1}} z_{\ell i_{2} t_{2}}$ is correlated with $z_{\ell i_{3} t_{3}} z_{\ell i_{4} t_{4}}$ for only finite terms of $\left(i_{3}, t_{3}\right)$ and $\left(i_{4}, t_{4}\right)$. It is noteworthy that Assumption 3.6 (iii) allows for individual dependence among finite neighbors and time dependence up to finite lags, which makes it weaker than the independence assumption of regressors across individuals in Su and Yang (2015).

Recall that $\boldsymbol{\zeta}=\left(\boldsymbol{\phi}^{\prime}, \sigma_{\varepsilon}^{2}, \boldsymbol{\delta}^{\prime}\right)^{\prime}$ and $\widehat{\boldsymbol{\zeta}}=\left(\widehat{\boldsymbol{\phi}}^{\prime}(\widehat{\boldsymbol{\delta}}), \widehat{\sigma}_{\varepsilon}^{2}(\widehat{\boldsymbol{\delta}}), \widehat{\boldsymbol{\delta}}^{\prime}\right)^{\prime}$. To show the consistency of QMLE $\widehat{\boldsymbol{\zeta}}$, we first verify that $\boldsymbol{\zeta}_{0}=\left(\boldsymbol{\phi}_{0}^{\prime}, \sigma_{\varepsilon 0}^{2}, \boldsymbol{\delta}_{0}^{\prime}\right)^{\prime}$ is identifiable. For the quasi-log-likelihood function at (3.2), given $\boldsymbol{\delta}$, its expectation $E\left[\ln \mathcal{L}_{N T}(\boldsymbol{\zeta})\right]$ is maximized at

$$
\widetilde{\boldsymbol{\phi}}(\boldsymbol{\delta})=\left[E\left(\widetilde{Z}^{\prime} \Omega_{N T}^{-1}\left(\boldsymbol{\beta}^{\star}\right) \widetilde{Z}\right)\right]^{-1} E\left(\widetilde{Z}^{\prime} \Omega_{N T}^{-1}\left(\boldsymbol{\beta}^{\star}\right) S_{N T}(\lambda) \boldsymbol{Y}\right)
$$


and

$$
\widetilde{\sigma}_{\varepsilon}^{2}(\boldsymbol{\delta})=\frac{1}{N T} E\left(\widetilde{\boldsymbol{V}}_{N T}^{\prime}(\boldsymbol{\delta}) \Omega_{N T}^{-1}\left(\boldsymbol{\beta}^{\star}\right) \tilde{\boldsymbol{V}}_{N T}(\boldsymbol{\delta})\right)
$$

where $\widetilde{\boldsymbol{V}}_{N T}(\boldsymbol{\delta})=S_{N T}(\lambda) \boldsymbol{Y}-\widetilde{Z} \widetilde{\boldsymbol{\phi}}(\boldsymbol{\delta})$. Define $\ln \ell_{N T}^{\star}(\boldsymbol{\delta})=\max _{\boldsymbol{\phi}, \sigma_{\varepsilon}^{2}} E\left[\ln \mathcal{L}_{N T}(\boldsymbol{\zeta})\right]$, then by $(3.7)$ and (3.8), we have

$$
\ln \ell_{N T}^{\star}(\boldsymbol{\delta})=-\frac{N T}{2}(\ln (2 \pi)+1)-\frac{N T}{2} \ln \widetilde{\sigma}_{\varepsilon}^{2}(\boldsymbol{\delta})-\frac{1}{2} \ln \left|\Omega_{N T}\left(\boldsymbol{\beta}^{\star}\right)\right|+\ln \left|S_{N T}(\lambda)\right| .
$$

Denote $\Omega_{0 N T}=\Omega_{N T}\left(\boldsymbol{\beta}_{0}^{\star}\right)$, since $E\left(\widetilde{Z}^{\prime} \Omega_{0 N T}^{-1} \boldsymbol{V}_{N T}\right)=0$ by Lemma 8 in the Supplementary Material, it can be shown that $\widetilde{\boldsymbol{\phi}}\left(\boldsymbol{\delta}_{0}\right)=\boldsymbol{\phi}_{0}+\left[E\left(\widetilde{Z}^{\prime} \Omega_{0 N T}^{-1} \widetilde{Z}\right)\right]^{-1} E\left(\widetilde{Z}^{\prime} \Omega_{0 N T}^{-1} \boldsymbol{V}_{N T}\right)=\boldsymbol{\phi}_{0}$. Then it follows that $\widetilde{\boldsymbol{V}}_{N T}\left(\boldsymbol{\delta}_{0}\right)=\boldsymbol{V}_{N T}$ and $\widetilde{\sigma}_{\varepsilon}^{2}\left(\boldsymbol{\delta}_{0}\right)=\sigma_{\varepsilon 0}^{2}$. Identification of $\boldsymbol{\delta}_{0}$ can be based on the maximum values of $\frac{1}{N T} \ln \ell_{N T}^{\star}(\boldsymbol{\delta})$, and hence that of $\phi_{0}$ and $\sigma_{\varepsilon 0}^{2}$ follows. The following condition is imposed for identification.

Assumption 3.7. For any $\boldsymbol{\delta} \neq \boldsymbol{\delta}_{0}$, it holds that

$$
\lim _{N \rightarrow \infty} \frac{1}{N T}\left[\ln \left|\widetilde{\sigma}_{\varepsilon}^{-2}(\boldsymbol{\delta}) \Omega_{N T}^{-1}\left(\boldsymbol{\beta}^{\star}\right) S_{N T}(\lambda)\right|-\ln \left|\sigma_{\varepsilon 0}^{-2} \Omega_{0 N T}^{-1} S_{0 N T}\right|\right] \neq 0
$$

Assumption 3.7 is equivalent to that $\lim _{N \rightarrow \infty} \frac{1}{N T}\left[\ln \ell_{N T}^{\star}(\boldsymbol{\delta})-\ln \ell_{N T}^{\star}\left(\boldsymbol{\delta}_{\mathbf{0}}\right)\right] \neq 0$ for any $\boldsymbol{\delta} \neq \boldsymbol{\delta}_{0}$, which ensures the identification of $\boldsymbol{\delta}_{0}$; see also Assumption 9 of Lee (2004) and Assumption $\mathrm{R}(i v)$ of Su and Yang (2015). Finally, the consistency of $\widehat{\boldsymbol{\delta}}$ holds by the identification and uniform convergence of $\frac{1}{N T}\left[\ln \ell_{N T}(\boldsymbol{\delta})-\ln \ell_{N T}^{\star}(\boldsymbol{\delta})\right]$ to zero on $\Delta$, and consequently the consistency of $\widehat{\phi}$ and $\widehat{\sigma}_{\varepsilon}^{2}$ follows.

Theorem 1. Under Assumptions 3.1, 3.4 - 3.7, then $\widehat{\boldsymbol{\zeta}} \rightarrow_{p} \boldsymbol{\zeta}_{0}$ as $N \rightarrow \infty$.

$$
\text { Let } \begin{aligned}
\Sigma & =\lim _{N \rightarrow \infty} \Sigma_{N} \text { and } \Sigma^{\star}=\lim _{N \rightarrow \infty} \Sigma_{N}^{\star} \text {, where } \Sigma_{N}=-E\left(\frac{1}{N T} \frac{\partial^{2} \ln \mathcal{L}_{N T}\left(\boldsymbol{\zeta}_{0}\right)}{\partial \boldsymbol{\zeta}_{\partial \boldsymbol{\zeta}^{\prime}}}\right) \text { and } \\
\Sigma_{N}^{\star} & =E\left(\frac{1}{\sqrt{N T}} \frac{\partial \ln \mathcal{L}_{N T}\left(\boldsymbol{\zeta}_{0}\right)}{\partial \boldsymbol{\zeta}} \frac{1}{\sqrt{N T}} \frac{\partial \ln \mathcal{L}_{N T}\left(\boldsymbol{\zeta}_{0}\right)}{\partial \boldsymbol{\zeta}^{\prime}}\right)+E\left(\frac{1}{N T} \frac{\partial^{2} \ln \mathcal{L}_{N T}\left(\boldsymbol{\zeta}_{0}\right)}{\partial \boldsymbol{\zeta} \partial \boldsymbol{\zeta}^{\prime}}\right) .
\end{aligned}
$$


By the consistency result given in Theorem 1 , the asymptotic normality of $\widehat{\zeta}$ can be obtained by Taylor expansion and the central limit theorem for linear-quadratic forms in Kelejian and Prucha (2001).

Theorem 2. Under Assumptions 3.2, 3.4- 3.7, if $\Sigma$ and $\Sigma^{\star}$ exist, and $\Sigma$ is positive definite, then $\sqrt{N T}\left(\widehat{\boldsymbol{\zeta}}-\boldsymbol{\zeta}_{0}\right) \rightarrow_{d} N\left(\mathbf{0}, \Sigma^{-1}+\Sigma^{-1} \Sigma^{\star} \Sigma^{-1}\right)$ as $N \rightarrow \infty$.

Theorem 2 provides the asymptotic distribution of the QMLE $\widehat{\boldsymbol{\zeta}}$, where the matrices $\Sigma_{N}$ and $\Sigma_{N}^{\star}$ are relegated to Section S3.1 of the Supplementary Material. If $\varepsilon_{i t}$ and $\eta_{i}$ are normally distributed, then the QMLE reduces to the MLE and its limiting distribution can be simplified to $\sqrt{N T}\left(\widehat{\boldsymbol{\zeta}}-\boldsymbol{\zeta}_{0}\right) \rightarrow_{d} N\left(\mathbf{0}, \Sigma^{-1}\right)$ as $N \rightarrow \infty$. Note that Theorems 11 and 2 allow $T$ to be fixed or tend to infinity, which makes QMLE applicable to both the short or long panel data.

To calculate the covariance matrix of $\widehat{\zeta}$ in practice, consistent estimates of $\Sigma$ and $\Sigma^{\star}$ are necessary. Obviously, the matrix $\Sigma=\lim _{N \rightarrow \infty}-E\left(\frac{1}{N T} \frac{\partial^{2} \ln \mathcal{L}_{N T}\left(\boldsymbol{\zeta}_{0}\right)}{\partial \boldsymbol{\zeta} \partial \boldsymbol{\zeta}^{\prime}}\right)$ can be consistently estimated by $\widehat{\Sigma}=-\frac{1}{N T} \frac{\partial^{2} \ln \mathcal{L}_{N T}(\widehat{\boldsymbol{\zeta}})}{\partial \boldsymbol{\zeta} \partial \widehat{\zeta}^{\prime}}$. However, for the following matrix

$$
\Gamma=\Sigma^{\star}+\Sigma=\lim _{N \rightarrow \infty} E\left(\frac{1}{\sqrt{N T}} \frac{\partial \ln \mathcal{L}_{N T}\left(\boldsymbol{\zeta}_{0}\right)}{\partial \boldsymbol{\zeta}} \frac{1}{\sqrt{N T}} \frac{\partial \ln \mathcal{L}_{N T}\left(\boldsymbol{\zeta}_{0}\right)}{\partial \boldsymbol{\zeta}^{\prime}}\right)
$$

its closed-form estimate is not readily available; see also Su and Yang (2015). Alternatively, we resort to the residual-based bootstrap and estimate $\Gamma$ by its bootstrap estimate $\widehat{\Gamma}_{B}$; see its detailed procedure and validity in the Supplementary Material. Consequently, the asymptotic covariance matrix of $\widehat{\zeta}$ can be estimated by $\widehat{\Sigma}^{-1} \widehat{\Gamma}_{B} \widehat{\Sigma}^{-1}$.

To estimate $\boldsymbol{\beta}_{0}=\left(\beta_{00}, \beta_{10}, \ldots, \beta_{p 0}\right)^{\prime}$ in model $(2.2)$, the constraint $\beta_{00} \equiv 1$ should be imposed for identification of $\boldsymbol{\beta}_{0}$ as in Koenker and Zhao (1994). Note that $\boldsymbol{\beta}_{0}^{\star}=\boldsymbol{\beta}_{0} \sigma_{\eta 0} / \sigma_{\varepsilon 0}$, we have $\beta_{\ell 0}=\beta_{\ell 0} / \beta_{00}=\beta_{\ell 0}^{\star} / \beta_{00}^{\star}$ for $\ell=1, \ldots, p$. Then based on the QMLE $\widehat{\boldsymbol{\zeta}}=\left(\widehat{\boldsymbol{\phi}}^{\prime}, \widehat{\sigma}_{\varepsilon}^{2}, \widehat{\lambda}, \widehat{\boldsymbol{\beta}}^{\star \prime}\right)^{\prime}$ 
with $\widehat{\boldsymbol{\beta}}^{\star}=\left(\widehat{\beta}_{0}^{\star}, \widehat{\beta}_{1}^{\star}, \ldots, \widehat{\beta}_{p}^{\star}\right)^{\prime}$, we can construct a consistent estimator for $\boldsymbol{\beta}_{0}$, denoted by $\check{\boldsymbol{\beta}}=\left(1, \check{\beta}_{1}, \ldots, \check{\beta}_{p}\right)^{\prime}$, where

$$
\check{\beta}_{\ell}=\widehat{\beta}_{\ell}^{\star} / \widehat{\beta}_{0}^{\star}, \quad \ell=1, \ldots, p .
$$

Under the conditions of Theorem 2, by the delta method (Doob, 1935), we can show that $\sqrt{N T}\left(\check{\beta}_{\ell}-\beta_{\ell 0}\right) \rightarrow_{d} N\left(0, \nabla h_{\ell}\left(\boldsymbol{\zeta}_{0}\right)^{\prime} \Sigma_{\zeta} \nabla h_{\ell}\left(\boldsymbol{\zeta}_{0}\right)\right)$ as $N \rightarrow \infty$, where $\Sigma_{\zeta}=\Sigma^{-1}+\Sigma^{-1} \Sigma^{\star} \Sigma^{-1}$ and $\nabla h_{\ell}(\boldsymbol{\zeta})=\partial h_{\ell}(\boldsymbol{\zeta}) / \partial \boldsymbol{\zeta}=\left(\mathbf{0}_{4+p+q}^{\prime},-\beta_{\ell}^{\star} / \beta_{0}^{\star 2}, \mathbf{0}_{\ell-1}^{\prime}, 1 / \beta_{0}^{\star}, \mathbf{0}_{p-1}^{\prime}\right)^{\prime}$ with $h_{\ell}(\boldsymbol{\zeta})=\beta_{\ell}^{\star} / \beta_{0}^{\star}$. And we can calculate the asymptotic variance of $\check{\beta}_{\ell}$ in practice by plugging in $\nabla h_{\ell}(\widehat{\boldsymbol{\zeta}})$ and $\widehat{\Sigma}_{\zeta}=$ $\widehat{\Sigma}^{-1} \widehat{\Gamma}_{B} \widehat{\Sigma}^{-1}$. However, $\check{\boldsymbol{\beta}}$ performs very poorly when the sample size is small or even moderate; see details in Table 3. As a result, we propose another estimator for $\boldsymbol{\beta}_{0}$ in Section 3.2.2, which is much more efficient for small and moderate samples.

\subsection{Conditional Quantile Estimation}

In Section 3.2.1, we first investigate the conditional quantile estimation for $\vartheta_{i}$ 's. Then we construct an efficient estimator for the conditional scale coefficient $\boldsymbol{\beta}$ of the random effects by optimally combing information over multiple quantile levels $\tau$ 's in Section 3.2 .2 .

\subsubsection{Weighted conditional quantile estimation}

Since the scale effect $\vartheta_{i}$ in model (3.1) is unobservable, to study its conditional quantiles, we first consider to obtain its observable approximate using the QMLE $\widehat{\zeta}$ from the first stage. Replacing $\boldsymbol{\zeta}$ by $\widehat{\boldsymbol{\zeta}}$ in model $(2.3)$, one can get $T$ random approximates of $\vartheta_{i}$ for each $i$. To fully make use of the information among these approximates, a natural way is to take the average of $T$ random approximates as the final approximate for $\vartheta_{i}$. Therefore, the pseudo 
scale effect denoted by $\widehat{\boldsymbol{\vartheta}}$, that is the approximate of $\boldsymbol{\vartheta}$, is given by

$$
\widehat{\boldsymbol{\vartheta}}=\left(\widehat{\vartheta}_{1}, \ldots, \widehat{\vartheta}_{N}\right)^{\prime}=\frac{1}{T} \sum_{t=1}^{T}\left[B_{N}(\widehat{\lambda}) \boldsymbol{y}_{t}-\widehat{\alpha} \boldsymbol{y}_{t-1}-Z_{t} \widehat{\gamma}-X \widehat{\boldsymbol{\psi}}\right]
$$

Thus, the pseudo random effect follows by $\widehat{\boldsymbol{\theta}}=X \widehat{\boldsymbol{\psi}}+\widehat{\boldsymbol{\vartheta}}$. Next the conditional quantile estimation can be applied to model (3.1) with the latent variable $\boldsymbol{\vartheta}$ replaced by its observable pseudo scale effect $\widehat{\vartheta}$. Due to the presence of conditional heteroscedasticity in $\vartheta_{i}$, we consider the WCQE given by

$$
\widehat{\boldsymbol{\varphi}}(\tau)=\left(\widehat{\varphi}_{0}(\tau), \widehat{\varphi}_{1}(\tau), \ldots, \widehat{\varphi}_{p}(\tau)\right)^{\prime}=\underset{\boldsymbol{\varphi} \in \Phi}{\operatorname{argmin}} \sum_{i=1}^{N} \frac{1}{\boldsymbol{x}_{a i}^{\prime} \widetilde{\boldsymbol{\beta}}_{c}} \rho_{\tau}\left(\widehat{\vartheta}_{i}-\boldsymbol{x}_{a i}^{\prime} \boldsymbol{\varphi}\right), \quad \tau \in \mathcal{T}
$$

where $\Phi$ is the parameter space of $\boldsymbol{\varphi}(\tau), \mathcal{T}$ is a closed subinterval of $(0,1), \rho_{\tau}(u)=$ $u(\tau-I(u<0))$ is the check function, and $\widetilde{\boldsymbol{\beta}}_{c}$ is a consistent estimator of $\boldsymbol{\beta}_{0}$ such that $\boldsymbol{x}_{a i}^{\prime} \widetilde{\boldsymbol{\beta}}_{c}$ is bounded away from zero for all $1 \leq i \leq N$; see also Koenker and Zhao (1994) and Zhao and Xiao (2014). For the choice of $\widetilde{\boldsymbol{\beta}}_{c}$, see more details in Remark 3 .

Theorem 3. Under Assumptions 3.3-3.7, as $N \rightarrow \infty$, then the following results hold,

(i) $\widehat{\vartheta}_{i}-\vartheta_{i}=o_{p}(1)$ for $1 \leq i \leq N$;

(ii) $\frac{1}{\sqrt{N}} \sum_{i=1}^{N}\left(\widehat{\vartheta}_{i}-\vartheta_{i}\right)=o_{p}(1)$.

Theorem 3 plays an important role in verifying the validity of the proposed hybrid estimation procedure. Specifically, Theorem $3(i)$ guarantees that replacing $\boldsymbol{\vartheta}$ with $\widehat{\vartheta}$ will not affect the theoretical properties of the WCQE $\widehat{\varphi}(\tau)$, and its proof requires $T \rightarrow \infty$ which is implied by $N \rightarrow \infty$ and Assumption 3.3. Theorem 3( $i i)$ largely simplifies the derivation of the Bahadur representation for $\widehat{\varphi}(\tau)$ below; see details in equation (S5.16) of the Supplementary Material. 
Denote the true value of $\boldsymbol{\varphi}(\tau)$ as $\boldsymbol{\varphi}_{0}(\tau)=\left(\varphi_{00}(\tau), \varphi_{10}(\tau), \ldots, \varphi_{p 0}(\tau)\right)^{\prime}$. Assume that the parameter space $\Phi$ is compact and $\boldsymbol{\varphi}_{0}(\tau)$ is its interior point; see also Assumption R2 of Chernozhukov and Hansen (2006) and Assumption 4.1 (b) of Canay (2011). To establish the theoretical properties of $\widehat{\varphi}(\tau)$, we need the following basic assumptions.

Assumption 3.8. For $1 \leq i \leq N, \vartheta_{i} \in \mathbb{R}$ has a bounded conditional density a.s., that is, $\sup _{\vartheta \in \mathbb{R}} f_{\vartheta_{i} \mid \boldsymbol{x}_{i}}(\vartheta)<K$ for some $K>0$ a.s., where $\mathbb{R}=(-\infty, \infty)$, and $f_{\vartheta_{i} \mid \boldsymbol{x}_{i}}(\vartheta)$ is bounded away from zero at the point $\boldsymbol{x}_{i}^{\prime} \varphi_{0}(\tau)$.

Assumption 3.9. For the function $\boldsymbol{\Pi}_{i}\left(\tau, \boldsymbol{\varphi}, r_{i}\right)=E\left[\frac{1}{\boldsymbol{x}_{a i}^{\prime} \boldsymbol{\beta}}\left(\tau-I\left(\vartheta_{i}<\boldsymbol{x}_{a i}^{\prime} \boldsymbol{\varphi}-r_{i}\right)\right) \boldsymbol{x}_{a i}\right]$ with $\left(\tau, \boldsymbol{\varphi}, r_{i}\right) \in \mathcal{T} \times \Phi \times \mathbb{R}$, the Jacobian matrices $\frac{\partial}{\partial \boldsymbol{\varphi}^{\prime}} \boldsymbol{\Pi}_{i}\left(\tau, \boldsymbol{\varphi}, r_{i}\right)$ and $\frac{\partial}{\partial r_{i}} \boldsymbol{\Pi}_{i}\left(\tau, \boldsymbol{\varphi}, r_{i}\right)$ for each $1 \leq i \leq N$ are continuous and have full rank, uniformly over $\mathcal{T} \times \Phi \times \mathbb{R}$.

Assumption 3.8 is needed to establish the uniform convergence of $\widehat{\varphi}(\tau)$, and this together with Assumption 3.9 is used for weak convergence of $\widehat{\varphi}(\tau)$; see Assumption R3 of Chernozhukov and Hansen (2006) and Assumption 4.1(c) of Canay (2011).

Theorem 4. Suppose the conditions of Theorem 3 hold. Then under Assumption 3.8, $\sup _{\tau \in \mathcal{T}}\left\|\widehat{\varphi}(\tau)-\varphi_{0}(\tau)\right\| \rightarrow_{p} 0$ as $N \rightarrow \infty$

Theorem 4 together with Theorems 1 and 3 , ensures that the conditional quantile of the random effect $\boldsymbol{\theta}$ can be consistently estimated by

$$
\widehat{Q}_{\boldsymbol{\theta}}(\tau \mid X)=X \widehat{\boldsymbol{\psi}}+X_{a} \widehat{\boldsymbol{\varphi}}(\tau)
$$

Denote the density function of $\eta_{i}$ by $f_{\eta}(\cdot)$. Let $S\left(\tau, \tau^{\prime}\right)=\min \left\{\tau, \tau^{\prime}\right\}-\tau \tau^{\prime}$ and $\Xi(\tau)=$ $f_{\eta}\left(Q_{\eta}(\tau)\right)$. Define the $(p+1) \times(p+1)$ matrices

$$
D_{N}=\frac{1}{N} \sum_{i=1}^{N} \frac{\boldsymbol{x}_{a i} \boldsymbol{x}_{a i}^{\prime}}{\left(\boldsymbol{x}_{a i}^{\prime} \boldsymbol{\beta}_{0}\right)^{2}}, D_{0}=\lim _{N \rightarrow \infty} D_{N}, \text { and } D_{1 N}(\tau)=\Xi(\tau) D_{N} .
$$


Theorem 5. Suppose the conditions of Theorem 4 hold. If $D_{0}$ exists and is positive definite, then under Assumption 3.9, as $N \rightarrow \infty$,

$$
\sqrt{N}\left(\widehat{\varphi}(\cdot)-\boldsymbol{\varphi}_{0}(\cdot)\right)=D_{1 N}^{-1}(\cdot) \mathbb{G}_{N}\left(\frac{\boldsymbol{x}_{a i}}{\boldsymbol{x}_{a i}^{\prime} \boldsymbol{\beta}_{0}} \Psi \cdot\left(\eta_{i}-Q_{\eta}(\cdot)\right)\right)+o_{p}(1) \rightsquigarrow \mathbb{G}(\cdot) \text { in } \ell^{\infty}(\mathcal{T})
$$

where $\Psi_{\tau}(u)=\tau-I(u<0)$, and $\mathbb{G}(\cdot)$ is a zero mean Gaussian process with covariance kernel $\Xi^{-1}(\tau) S\left(\tau, \tau^{\prime}\right) \Xi^{-1}\left(\tau^{\prime}\right) D_{0}^{-1}$.

Theorem 5 states the weak convergence of the quantile regression process $\widehat{\varphi}(\cdot)$. For any fixed quantile level $\tau$, by Theorem 5, it follows that, as $N \rightarrow \infty$,

$$
\sqrt{N}\left(\widehat{\varphi}(\tau)-\boldsymbol{\varphi}_{0}(\tau)\right) \rightarrow_{d} N\left(\mathbf{0}, \tau(1-\tau) \Xi^{-2}(\tau) D_{0}^{-1}\right)
$$

To calculate the covariance matrix of $\widehat{\varphi}(\tau)$ in practice, consistent estimators of $\Xi(\tau)=$ $f_{\eta}\left(Q_{\eta}(\tau)\right)$ and $D_{0}$ are required. Define the error function $\widehat{\eta}_{i}(\boldsymbol{\beta})=\widehat{\vartheta}_{i} /\left(\boldsymbol{x}_{a i}^{\prime} \boldsymbol{\beta}\right)$, then the residuals can be computed by $\widetilde{\eta}_{i}=\widehat{\eta}_{i}\left(\widetilde{\boldsymbol{\beta}}_{c}\right)$, where $\widetilde{\boldsymbol{\beta}}_{c}$ is a consistent estimate of $\boldsymbol{\beta}_{0}$ as in (3.12). The density function $f_{\eta}(\cdot)$ can be estimated by the kernel density estimator $\tilde{f}_{\eta}(x)=$ $(N h)^{-1} \sum_{i=1}^{N} K\left(\left(x-\widetilde{\eta}_{i}\right) / h\right)$, where $K(\cdot)$ is the kernel function and $h$ is the bandwidth. In practice, we suggest using the Gaussian kernel for $K(\cdot)$ and its rule-of-thumb bandwidth, $h=$ $0.9 N^{-1 / 5} \min \{s, \widehat{R} / 1.34\}$, where $s$ and $\widehat{R}$ are the sample standard deviation and interquartile of the residuals, respectively; see also Zhao and Xiao (2014). As a result, $\Xi(\tau)$ can be estimated by $\tilde{f}_{\eta}\left(\bar{Q}_{\eta}(\tau)\right)$, where $\bar{Q}_{\eta}(\tau)$ is the sample $\tau$-th quantile of $\left\{\widetilde{\eta}_{i}\right\}_{i=1}^{N}$. Moreover, $D_{0}$ can be approximated by $D_{N}$ with $\boldsymbol{\beta}_{0}$ replaced by $\widetilde{\boldsymbol{\beta}}_{c}$, i.e. $\widetilde{D}_{N}=\frac{1}{N} \sum_{i=1}^{N} \frac{\boldsymbol{x}_{a i} \boldsymbol{x}_{a i}^{\prime}}{\left(\boldsymbol{x}_{a i}^{\prime} \widetilde{\boldsymbol{\beta}}_{c}\right)^{2}}$. Consequently, the asymptotic covariance matrix of $\widehat{\boldsymbol{\varphi}}(\tau)$ can be estimated by plugging in $\bar{\Xi}(\tau)=\widetilde{f}_{\eta}\left(\bar{Q}_{\eta}(\tau)\right)$ and $\widetilde{D}_{N}$ to replace $\Xi(\tau)$ and $D_{0}$, respectively.

Remark 1. (Discussion on the estimation of $\boldsymbol{\psi}$ ) As the conditional mean coefficient of random effects, $\boldsymbol{\psi}$ can be estimated either by the quasi-maximum likelihood estimation in the 
first stage or the conditional quantile estimation for model $Q_{\boldsymbol{\theta}}(\tau \mid \boldsymbol{X})=\boldsymbol{X} \boldsymbol{\psi}+\boldsymbol{X}_{a} \boldsymbol{\varphi}(\tau)$ in the second stage. However, by comparing Theorems and 5, it can be found that the QMLE $\widehat{\boldsymbol{\psi}}$ of $\boldsymbol{\psi}$ has faster convergence rate of $\sqrt{N T}$ than that of $\sqrt{N}$ for the quantile estimator obtained using model $Q_{\boldsymbol{\theta}}(\tau \mid \boldsymbol{X})=\boldsymbol{X} \boldsymbol{\psi}+\boldsymbol{X}_{a} \boldsymbol{\varphi}(\tau)$. This is another reason that the proposed two-stage hybrid estimation procedure does not consider the quantile estimation for both $\boldsymbol{\psi}$ and $\varphi(\tau)$ simultaneously.

Remark 2. (Discussion on an alternative estimation procedure) For estimation of DSAR model (2.1) with the random effect $\theta_{i}$ specified by (2.2), we can consider the following alternative estimation procedure.

Stage 1': Estimate $\left(\alpha, \gamma^{\prime}, \sigma_{\varepsilon}^{2}, \boldsymbol{\delta}\right)^{\prime}$ by QMLE using the transformation approach or firstdifferencing to eliminate individual effects $\theta_{i}$ 's (Lee and $Y u$, 2010; Yu et al., 2008).

Stage 2': Define the pseudo random effect $\widetilde{\boldsymbol{\theta}}$ similarly as in (3.11). Then apply the WCQE to model (2.4) with $\boldsymbol{\theta}$ replaced by its approximate $\widetilde{\boldsymbol{\theta}}$. And $\tau$-independent $\boldsymbol{\psi}$ and $\boldsymbol{\beta}$ can be estimated by $\widetilde{\boldsymbol{\psi}}$ and $\widetilde{\boldsymbol{\beta}}$ via combining the information of quantile estimators at multiple quantile levels, respectively.

Note that the transformation approach or first-differencing in Stage 1'ignores the structure of $\boldsymbol{\theta}$ in (2.2), which makes the estimation less efficient. Moreover, $\widetilde{\boldsymbol{\psi}}$ obtained by quantile regression in Stage 2' will also result in efficiency loss as discussed in Remark 1. Thus we prefer the two-stage hybrid estimation procedure proposed in Section 3 .

Remark 3. (Choice of $\widetilde{\boldsymbol{\beta}}_{c}$ in (3.12) for the WCQE) To conduct the weighted quantile re-

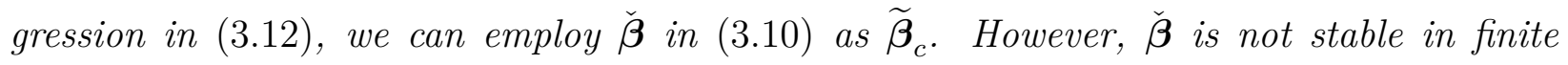
sample cases, so we construct an alternative choice for $\widetilde{\boldsymbol{\beta}}_{c}$. Note that $\boldsymbol{\varphi}_{0}(\tau)=Q_{\eta}(\tau) \boldsymbol{\beta}_{0}$, we can construct $\widetilde{\boldsymbol{\beta}}_{c}$ by combining the information of the estimator for $\boldsymbol{\varphi}_{0}(\tau)$ over multi- 
ple quantile levels. Furthermore, $\varphi_{0}(\tau)$ and $Q_{\eta}(\tau)$ have the same sign, thus we consider $\sum_{k=1}^{K}\left|\boldsymbol{\varphi}_{0}\left(\tau_{k}\right)\right|=\boldsymbol{\beta}_{0} \sum_{k=1}^{K}\left|Q_{\eta}\left(\tau_{k}\right)\right|$ alternatively to avoid the offset among positive and negative values for both terms at different quantile levels. Following Koenker and Zhao (1994), we adopt the constraint $\beta_{00} \equiv 1$ to ensure the identification of $\boldsymbol{\beta}_{0}$ and $Q_{\eta}(\tau)$. As a result, we suggest a choice for $\widetilde{\boldsymbol{\beta}}_{c}$ in $(3.12)$ as follows

$$
\widetilde{\boldsymbol{\beta}}_{c}=\frac{\sum_{k=1}^{K}\left|\widetilde{\boldsymbol{\varphi}}\left(\tau_{k}\right)\right|}{\sum_{k=1}^{K}\left|\widetilde{Q}_{\eta}\left(\tau_{k}\right)\right|}
$$

where $\tilde{\boldsymbol{\varphi}}\left(\tau_{k}\right)$ 's with $\tau_{k}=k /(K+1)$ for $1 \leq k \leq K$, are the unweighted conditional quantile estimators defined by $\widetilde{\boldsymbol{\varphi}}\left(\tau_{k}\right)=\left(\widetilde{\varphi}_{0}\left(\tau_{k}\right), \ldots, \widetilde{\varphi}_{p}\left(\tau_{k}\right)\right)^{\prime}=\operatorname{argmin}_{\boldsymbol{\varphi} \in \Phi} \sum_{i=1}^{N} \rho_{\tau_{k}}\left(\widehat{\vartheta}_{i}-\boldsymbol{x}_{a i}^{\prime} \boldsymbol{\varphi}\right)$ and $\widetilde{Q}_{\eta}\left(\tau_{k}\right)=\widetilde{\varphi}_{0}\left(\tau_{k}\right)$; see also Zhu et al. (2018). Clearly, (3.15) guarantees the positivity of $\widetilde{\boldsymbol{\beta}}_{c}$.

\subsubsection{Weighted quantile average estimation}

For the conditional scale coefficient $\boldsymbol{\beta}$ of the random effects, we have proposed two consistent estimators $\check{\boldsymbol{\beta}}$ and $\widetilde{\boldsymbol{\beta}}_{c}$ in $(3.10)$ and $(3.15)$, respectively. However, $\check{\boldsymbol{\beta}}$ is not suggested as it is not stable in small or even moderate samples. For $\widetilde{\boldsymbol{\beta}}_{c}$, it is actually an equally weighted quantile average estimator by assigning equal weight $\left[\sum_{k=1}^{K}\left|\widetilde{Q}_{\eta}\left(\tau_{k}\right)\right|\right]^{-1}$ to the WCQE $\widehat{\varphi}(\cdot)$ at each quantile level, which may lead to a less efficient estimator. We next introduce an efficient estimator of $\boldsymbol{\beta}$ by optimally combining the information of $\widehat{\varphi}(\cdot)$ across $K$ quantile levels: $\tau_{k}=k /(K+1)$ for $1 \leq k \leq K$, where $K$ is a fixed integer.

Recall that $\boldsymbol{\beta}_{0}=\left(\beta_{00}, \beta_{10}, \ldots, \beta_{p 0}\right)^{\prime}$ is the true value of $\boldsymbol{\beta}$, and $\boldsymbol{\varphi}_{0}(\tau)=Q_{\eta}(\tau) \boldsymbol{\beta}_{0}$ with $\varphi_{\ell 0}(\tau)=Q_{\eta}(\tau) \beta_{\ell 0}$ holds for $\ell=0,1, \ldots, p$. Note that for any weight vector $\boldsymbol{\pi}_{K}=$ $\left(\pi_{1}, \ldots, \pi_{K}\right)^{\prime}$ satisfying $\sum_{k=1}^{K} \pi_{k} Q_{\eta}\left(\tau_{k}\right)=1$, it holds that $\boldsymbol{\beta}_{0}=\sum_{k=1}^{K} \pi_{k} \boldsymbol{\varphi}_{0}\left(\tau_{k}\right)$ under the constraint $\beta_{00} \equiv 1$. This motivates us to combine $\left\{\widehat{\boldsymbol{\varphi}}\left(\tau_{k}\right), 1 \leq k \leq K\right\}$ linearly to define the 
WQAE of $\boldsymbol{\beta}$ as follows

$$
\widehat{\boldsymbol{\beta}}\left(\boldsymbol{\pi}_{K}\right)=\sum_{k=1}^{K} \pi_{k} \widehat{\boldsymbol{\varphi}}\left(\tau_{k}\right) \quad \text { with } \quad \sum_{k=1}^{K} \pi_{k} Q_{\eta}\left(\tau_{k}\right)=1,
$$

see also Zhao and Xiao (2014).

Denote $\mathscr{B}$ as the parameter space of $\boldsymbol{\beta}$, and assume $\mathscr{B}$ is compact and $\boldsymbol{\beta}_{0}$ is an interior point. Define the $K \times K$ matrix $H=\left\{h_{i j}, 1 \leq i, j \leq K\right\}$ with $h_{i j}=\Xi^{-1}\left(\tau_{i}\right) S\left(\tau_{i}, \tau_{j}\right) \Xi^{-1}\left(\tau_{j}\right)$. The asymptotics of $\widehat{\boldsymbol{\beta}}\left(\boldsymbol{\pi}_{K}\right)$ and the optimal choice for $\boldsymbol{\pi}_{K}$ are provided below.

Theorem 6. Suppose the conditions of Theorem 5 hold, then as $N \rightarrow \infty$,

$$
\sqrt{N}\left(\widehat{\boldsymbol{\beta}}\left(\boldsymbol{\pi}_{K}\right)-\boldsymbol{\beta}_{0}\right) \rightarrow_{d} N\left(0, \Upsilon\left(\boldsymbol{\pi}_{K}\right)\right)
$$

where $\Upsilon\left(\boldsymbol{\pi}_{K}\right)=\boldsymbol{\pi}_{K}^{\prime} H \boldsymbol{\pi}_{K} D_{0}^{-1}$. Moreover, the optimal weight is

$$
\boldsymbol{\pi}_{o p t, K}=\underset{\boldsymbol{\pi}, \text { s.t. } \boldsymbol{\pi}^{\prime} \boldsymbol{q}=1}{\operatorname{argmin}} \Upsilon\left(\boldsymbol{\pi}_{K}\right)=\frac{H^{-1} \boldsymbol{q}}{\boldsymbol{q}^{\prime} H^{-1} \boldsymbol{q}},
$$

where $\boldsymbol{q}=\left(Q_{\eta}\left(\tau_{1}\right), \ldots, Q_{\eta}\left(\tau_{K}\right)\right)^{\prime}$. Thus the asymptotic covariance of the optimal WQAE $\widehat{\boldsymbol{\beta}}\left(\boldsymbol{\pi}_{o p t, K}\right)$ is $\Upsilon\left(\boldsymbol{\pi}_{o p t, K}\right)=\left(\boldsymbol{q}^{\prime} H^{-1} \boldsymbol{q}\right)^{-1} D_{0}^{-1}$.

To estimate the optimal weight $\boldsymbol{\pi}_{\text {opt,K}}$, consistent estimators of $\boldsymbol{q}$ and $H$ are necessary. We can approximate $\boldsymbol{q}$ by $\widehat{\boldsymbol{q}}=\left(\widehat{Q}_{\eta}\left(\tau_{1}\right), \ldots, \widehat{Q}_{\eta}\left(\tau_{K}\right)\right)^{\prime}$ with $\widehat{Q}_{\eta}(\tau)=\widehat{\varphi}_{0}(\tau)$, and estimate $H$ by $\widehat{H}=\left\{\widehat{h}_{i j}, 1 \leq i, j \leq K\right\}$ with $\widehat{h}_{i j}=\widehat{\Xi}^{-1}\left(\tau_{i}\right) S\left(\tau_{i}, \tau_{j}\right) \widehat{\Xi}^{-1}\left(\tau_{j}\right)$ and $\widehat{\Xi}(\tau)=\widetilde{f}_{\eta}\left(\widehat{Q}_{\eta}(\tau)\right)$. Here using $\widehat{Q}_{\eta}(\tau)$ instead of the empirical quantile $\bar{Q}_{\eta}(\tau)$ to estimate $\boldsymbol{q}$ can ensure the estimate of $\beta_{00}$ being one. Then a consistent estimator $\widehat{\boldsymbol{\pi}}_{\text {opt }, K}=\left(\widehat{\pi}_{1, \text { opt }}, \ldots, \widehat{\pi}_{K, \text { opt }}\right)^{\prime}$ of $\boldsymbol{\pi}_{\text {opt }, K}=$ $\left(\pi_{1, o p t}, \ldots, \pi_{K, \text { opt }}\right)^{\prime}$ can be obtained by plugging in $\widehat{H}$ and $\widehat{\boldsymbol{q}}$. Finally, the optimal WQAE can be calculated by $\widehat{\boldsymbol{\beta}}\left(\widehat{\boldsymbol{\pi}}_{o p t, K}\right)=\sum_{k=1}^{K} \widehat{\pi}_{k, \text { opt }} \widehat{\boldsymbol{\varphi}}\left(\tau_{k}\right)$. It can be shown that $\sqrt{N}\left(\widehat{\boldsymbol{\beta}}\left(\widehat{\boldsymbol{\pi}}_{o p t, K}\right)-\boldsymbol{\beta}_{0}\right) \rightarrow_{d}$ $N\left(0, \Upsilon\left(\boldsymbol{\pi}_{o p t, K}\right)\right)$ as $N \rightarrow \infty$, which implies that $\widehat{\boldsymbol{\beta}}\left(\widehat{\boldsymbol{\pi}}_{o p t, K}\right)$ and $\widehat{\boldsymbol{\beta}}\left(\boldsymbol{\pi}_{o p t, K}\right)$ have the same asymptotic efficiency. 
Remark 4. (Comparison of $\check{\boldsymbol{\beta}}, \widetilde{\boldsymbol{\beta}}_{c}$ and $\widehat{\boldsymbol{\beta}}\left(\widehat{\boldsymbol{\pi}}_{\text {opt }, K}\right)$ ) For $\check{\boldsymbol{\beta}}$ and $\widehat{\boldsymbol{\beta}}\left(\widehat{\boldsymbol{\pi}}_{\text {opt }, K}\right)$, they are estimated by different methods and thus their theoretical comparison is infeasible. Alternatively, we compare their finite sample performance by simulation study. Simulation results in Section 4 indicate that $\widehat{\boldsymbol{\beta}}\left(\widehat{\boldsymbol{\pi}}_{\text {opt }, K}\right)$ outperforms $\check{\boldsymbol{\beta}}$ in biases, empirical and asymptotic standard deviations when the sample size is small or even moderate. In contrast, notice that the convergence rates of $\widehat{\boldsymbol{\beta}}\left(\widehat{\boldsymbol{\pi}}_{\text {opt }, K}\right)$ and $\check{\boldsymbol{\beta}}$ are $\sqrt{N}$ and $\sqrt{N T}$, respectively, thus $\check{\boldsymbol{\beta}}$ can be more efficient than $\widehat{\boldsymbol{\beta}}\left(\widehat{\boldsymbol{\pi}}_{\text {opt }, K}\right)$ for large samples. We also compare the finite sample performance of $\widehat{\boldsymbol{\beta}}\left(\widehat{\boldsymbol{\pi}}_{\text {opt }, K}\right)$ and $\widetilde{\boldsymbol{\beta}}_{c}$, and simulation results show that $\widehat{\boldsymbol{\beta}}\left(\widehat{\boldsymbol{\pi}}_{\text {opt }, K}\right)$ has smaller biases and empirical standard deviations than $\widetilde{\boldsymbol{\beta}}_{c}$. Therefore, we suggest to improve efficiency using $\widehat{\boldsymbol{\beta}}\left(\widehat{\boldsymbol{\pi}}_{\text {opt }, K}\right)$ when sample size is small or moderate, whereas $\check{\boldsymbol{\beta}}$ is preferred in large sample settings owing to its faster convergence rate.

\section{Simulation Study}

This section conducts a simulation experiment to evaluate the finite sample performance of the proposed two-stage hybrid estimation procedure in Section 3, including the Gaussian QMLE $\widehat{\boldsymbol{\zeta}}$, the WCQE $\widehat{\boldsymbol{\varphi}}(\tau)$ and the WQAE $\widehat{\boldsymbol{\beta}}\left(\widehat{\boldsymbol{\pi}}_{o p t, K}\right)$.

The data are generated from the following model

$$
\left\{\begin{array}{l}
y_{i t}=\theta_{i}+0.5 y_{i, t-1}+0.5 \sum_{j=1}^{N} w_{N, i j} y_{j t}+z_{i t}+\varepsilon_{i t}, \\
\theta_{i}=1+x_{i}+\left(1+\left|x_{i}\right|\right) \eta_{i},
\end{array}\right.
$$

where the innovations $\left\{\varepsilon_{i t}\right\}$ are independently standard normal, $\left\{\eta_{i}\right\}$ are independently standard normal or follow the uniform distribution $U(-\sqrt{3}, \sqrt{3})$, and $\left\{x_{i}\right\}$ and $\left\{z_{i t}\right\}$ are independently generated randomly from the uniform distribution $U(-1,1)$ and standard normal distribution, respectively. The spatial weights matrix is generated according to Rook con- 
tiguity and row normalized (Yu et al., 2008; Su and Yang, 2015). Note that the QMLE at Stage 1 has $\sqrt{N T}$-convergence rate under Assumption 3.2, which allows two cases: $(i)$ both $N$ and $T$ are large; $(i i) N$ is large but $T$ is fixed. Whereas both WCQE and WQAE at Stage 2 have $\sqrt{N}$-convergence rate under Assumption 3.3, which only allows case $(i)$. Hence, we consider three sample settings $(N, T)=(20,20),(20,50)$ and $(100,50)$ for QMLE, and $(N, T)=(20,20),(100,50)$ and $(300,200)$ for WCQE and WQAE, with 1000 replications generated for each sample setting.

We aim to estimate the parameters $\boldsymbol{\zeta}_{0}=\left(\alpha_{0}, \gamma_{10}, \psi_{00}, \psi_{10}, \sigma_{\varepsilon 0}^{2}, \lambda_{0}, \beta_{00}^{\star}, \beta_{10}^{\star}\right)^{\prime}, \boldsymbol{\varphi}_{0}(\tau)=$ $\left(\varphi_{00}(\tau), \varphi_{10}(\tau)\right)^{\prime}$ and $\beta_{10}$ using the QMLE $\widehat{\boldsymbol{\zeta}}$, the WCQE $\widehat{\boldsymbol{\varphi}}(\tau)$ and the WQAE $\widehat{\beta}_{1}\left(\widehat{\boldsymbol{\pi}}_{o p t, K}\right)$, respectively, where $\left(\beta_{00}^{\star}, \beta_{10}^{\star}\right)=\left(1, \beta_{10}\right) \sigma_{\eta 0} / \sigma_{\varepsilon 0}$ and $\boldsymbol{\varphi}_{0}(\tau)=\left(1, \beta_{10}\right) Q_{\eta}(\tau)$. The quasi-Newton method (Doob, 1935) is employed for solving the QMLE. For the WCQE, we use the R package "nloptr" (Johnson, 2021) with the same sign restriction imposed on each element of $\widehat{\boldsymbol{\varphi}}(\tau)$ owing to $\boldsymbol{\varphi}_{0}(\tau)=\boldsymbol{\beta}_{0} Q_{\eta}(\tau)$ and $\boldsymbol{\beta}_{0} \geq 0$. For the WQAE, we employ $K=9$ quantile levels $\tau_{k}=k / 10$ with $k=1, \ldots, 9$, and the resulting $\widehat{\beta}_{1}\left(\widehat{\boldsymbol{\pi}}_{o p t, K}\right)$ is almost nonnegative under the same sign restriction for $\widehat{\varphi}(\tau)$.

Table 1 reports the biases, empirical standard deviations (ESDs) and asymptotic standard deviations (ASDs) of the QMLE $\widehat{\boldsymbol{\zeta}}$ at Stage 1, where ASDs are calculated by the residualbased bootstrap with bootstrap sample size $B=500$. Recall that we require $N \rightarrow \infty$ theoretically, we only focus on the comparison of $(N, T)$ settings with $N$ increasing. Comparing the results of settings $(N, T)=(20,20)$ and $(100,50)$ in which $T$ increases at a slower rate than $N$, we can see that most of the biases, ESDs and ASDs become smaller as $N$ increases. We have the same finding when compares the results of setting $(N, T)=(20,50)$ with that of setting $(100,50)$ where $T$ is fixed as $N$ increases. Moreover, the biases for the 
normal distribution of $\eta_{i}$ are mostly smaller than those for the uniform distribution. This is as expected as the QMLE reduces to the MLE when $\eta_{i}$ 's are normally distributed.

Table 2 summarizes the biases, ESDs and ASDs of the WCQE $\widehat{\varphi}(\tau)$ at Stage 2 for three quantile levels $\tau=0.25,0.5$ and 0.75 , and three sample settings $(N, T)=(20,20),(100,50)$ and $(300,200)$. We have the following findings: (i) for both normally and uniformly distributed innovations $\left\{\eta_{i}\right\}$, as $N$ increases, most of the biases, ESDs and ASDs become smaller, and the ESDs get closer to their corresponding ASDs; (ii) the performance of $\widehat{\varphi}(\tau)$ gets better as the quantile level $\tau$ gets closer to the center. In addition, the ESDs are obviously smaller than the corresponding ASDs at $\tau=0.5$ because $Q_{\eta}(0.5) \equiv 0$ holds for symmetric distributions centered at zero and thus $\varphi_{00}(0.5)=\varphi_{10}(0.5) \equiv 0$, which makes ESDs abnormally small under the same sign constraint. However the ESDs are closer to their corresponding ASDs for larger $N$; see Table S.1 for evidence in the Supplementary Material.

Table 3 provides the biases, ESDs and ASDs of the WQAE $\widehat{\beta}_{1}\left(\widehat{\boldsymbol{\pi}}_{\text {opt }, K}\right)$ at Stage 2 for three sample settings $(N, T)=(20,20),(100,50)$ and $(300,200)$. For comparison, the results of $\check{\beta}_{1}$ in $(3.10)$ and $\widetilde{\beta}_{1 c}$ in $(3.15)$ are also reported, where the extra setting $(N, T)=(50,20)$ is added to show the performance of $\check{\beta}_{1}$ in cases with moderate sample sizes. It can be seen that, as $N$ increases, the biases, ESDs and ASDs of all estimators become smaller, and the ESDs get closer to the corresponding ASDs. Moreover, the ESDs of $\widehat{\beta}_{1}\left(\widehat{\boldsymbol{\pi}}_{o p t, K}\right)$ will move closer to their corresponding ASDs for larger $N$; see Table $S .2$ in the Supplementary Material. In addition, the estimate $\breve{\beta}_{1}$ is not satisfactory as the the sample size is not large, and $\widetilde{\beta}_{1 c}$ is usually less efficient than $\widehat{\beta}_{1}\left(\widehat{\boldsymbol{\pi}}_{o p t, K}\right)$ in terms of ESDs and ASDs.

In summary, the finite sample performance of the two-stage hybrid estimation procedure 
is reasonable, which validates the accuracy of the asymptotics in Section 3 .

\section{Empirical Analysis of Air Quality}

This section illustrates the usefulness of the proposed model and its estimation procedure by analysing the pure influence of socio-economic factors on the air quality in China after the spatial-temporal effects are eliminated.

We choose the air quality index (AQI) as the indicator for air quality, downloaded from China National Environmental Monitoring Centre (http://www.cnemc.cn/), which spans from January 1, 2018 to December 16, 2018 among 143 cities available in China. Since AQI is closely related to the meteorological factors (Yang et al., 2007; Lee et al., 2012), we select temperature (TEM), precipitation (PRE) and wind speed (WIN) to represent the exogenous spatial-temporal effects in our model, which is downloaded from National Meteorological Information Center (http://data.cma.cn/en). Moreover, AQI can also be affected by the socio-economic factors related to each city (Chen and Xu, 2017; Yu and Liu, 2020). Hence, we use Gross Regional Product (GRP) and secondary industry share in GRP (Industry) as the space-specific factors, and the data are obtained from China City Statistical Yearbook. Many references indicate that population or population density is also an important socioeconomic factor that can influence air quality, but Figure 1 shows that there is a significant positive correlation between GRP and population (or population density) in China, hence we only include GRP to avoid multicollinearity. To balance the size and information of data in time dimension, we transform the original average hourly data of AQI and average daily data of TEM and WIN into average weekly data by average, and transform the original cumulative daily data of PRE into cumulative weekly data by summation. Finally we obtain 
the spatial-temporal dataset with $N=143$ and $T=49$.

In Figure 2, the heat maps of the AQI of some local areas of China from the first to ninth weeks in 2018 imply that there are spatial and temporal effects in the air quality. This motivates us to consider the spatial and temporal effects with our model. In this study, we aim to understand the influence of the local socio-economic factors on the air quality with those spatial-temporal effects controlled so that the governments can evaluate how their local policies work in improving air qualities.

Note that the scales of AQI, TEM, PRE, WIN, GRP and Industry differ from each other as clearly demonstrated in Table 4, so we divide them by the estimated standard deviations, respectively. Moreover, we centralize TEM, PRE and WIN owing to the zero mean requirement by Assumption 3.6 (ii). In accordance with the model setting in (2.1), for $i=1, \ldots, 143$ and $t=0, \ldots, 49$, we denote the processed variables AQI, TEM, PRE, WIN, GRP and Industry as $\left\{y_{i t}\right\},\left\{z_{1 i t}\right\},\left\{z_{2 i t}\right\},\left\{z_{3 i t}\right\},\left\{x_{1 i}\right\}$ and $\left\{x_{2 i}\right\}$, respectively. We consider the proposed two-stage hybrid estimation procedure in Section 3. The fitted model of (2.1) is then given by

$$
y_{i t}=\widehat{\theta}_{i}+0.178 y_{i, t-1}+0.648 \sum_{j=1}^{N} w_{N, i j} y_{j t}-0.096 z_{1 i t}-0.065 z_{2 i t}-0.078 z_{3 i t}+\widehat{\varepsilon}_{i t},
$$

where the spatial weights matrix $W_{N}=\left\{w_{N, i j}=a_{i j} / d_{i}\right\}$ is a row-normalized binary adjacency matrix with $d_{i}=\sum_{j=1}^{N} a_{i j}, a_{i i}=0$ and $a_{i j}=1$ if the $i$-th city is adjacent to the $j$-th city geographically, otherwise $a_{i j}=0$ for $i \neq j$. The summary information of fitted coefficients in model (5.1) is provided in Table 5, where the standard errors are calculated based on the residual-based bootstrap with bootstrap sample size $B=500$. We observe that the coefficients are significantly nonzeros at the $10 \%$ significance level. The fitted model (5.1) indicates that the first lag of AQI and its neighbors have positive impacts on AQI, while the 
temperature, precipitation and wind speed have negative impacts on AQI. These findings are consistent with our common sense.

We are particularly interested in the influence of $x_{1 i}$ and $x_{2 i}$ on the low and high quantile levels of individual random effects $\theta_{i}$ 's, that is the influence of socio-economic factors on the air quality in good or poor status after the spatial-temporal effects are removed. For this purpose, we choose two quantile levels $\tau=0.25$ and 0.75 for illustration. The estimated conditional quantile functions of $\theta_{i}$ in model (2.4) are

$$
\left\{\begin{array}{l}
\widehat{Q}_{\theta_{i}}\left(0.25 \mid \boldsymbol{x}_{i}\right)=\left(0.171+0.078 x_{1 i}+0.063 x_{2 i}\right)+\left(-0.066-0.016\left|x_{1 i}\right|-0.046\left|x_{2 i}\right|\right), \\
\widehat{Q}_{\theta_{i}}\left(0.75 \mid \boldsymbol{x}_{i}\right)=\left(0.171+0.078 x_{1 i}+0.063 x_{2 i}\right)+\left(0.030+0.021\left|x_{1 i}\right|+0.014\left|x_{2 i}\right|\right),
\end{array}\right.
$$

where the summary information of fitted coefficients is reported in Table 5. For each equation of (5.2), the first parenthesis corresponds to the estimated conditional location of $\theta_{i}$ while the second parenthesis represents the conditional quantile estimate of the scale effect. Since the conditional location of $\theta_{i}$ is actually the conditional expectation of $\theta_{i}$, the fitted conditional expectation of $\theta_{i}$ is $\widehat{E}\left(\theta_{i} \mid \boldsymbol{x}_{i}\right)=0.171+0.078 x_{1 i}+0.063 x_{2 i}$. Notice that $x_{1 i}, x_{2 i}>0$ for all $i$ in our situation, then $\left|x_{\ell i}\right|$ equal to $x_{\ell i}$ for $\ell=1,2$. As a result, based on model (5.2) and Table 5, we have the following conclusions: (i) both the secondary industry share and GRP may impose positive impacts on the conditional mean of $\theta_{i}$ as well as its low and high quantiles; (ii) with the spatial-temporal effects eliminated, the insignificant positive impact on AQI of GRP implies that China may locate near the apex of the environmental Kuznets curve (Stern et al., 1996), that is, the adverse impact of China's economic development on the enviroment diminishes gradually; (iii) the secondary industry share has statistically significant positive impact on the conditional location of $\theta_{i}$ at $10 \%$ significance level, which indicates that the secondary industry may cause the deterioration of air quality in China. 
Moreover, the variable $x_{2 i}$ is significantly related with the conditional scale of $\theta_{i}$ at the lower quantile level while no significant effect is observed at the higher level under $10 \%$ significance level, which provides evidence of possible heterogeneity in AQI due to the secondary industry share.

\section{Conclusion and Discussion}

This paper introduces a dynamic spatial autoregressive model with heterogeneous random effects, which is useful in many cases with complicated correlation structures. Although we focus on the space-specific factors in this paper, this idea can be similarly used in other random effect models to account for possible heterogeneity based on quantile regression.

There are several potential research topics in the framework of the proposed new model. First, note that the asymptotic covariance of the QMLE in Theorem 2 is infeasible to compute, and the limiting distributions of the WCQE and WQAE in (3.14) and Theorem 6 are not convenient to estimate due to the unknown density function $f_{\eta}(\cdot)$ of innovations whose estimation needs some non-parametric methods with tuning parameters. The bootstrap method we used to approximate the covariance matrix of QMLE only introduces the perturbation into the individual level but ignores the time level, which can make it less efficient. As a result, we can consider a new method perturbing the information of individual and time levels simultaneously for the proposed model, and hopefully the bootstrapping can simplify the estimation of asymptotic distributions and lead to more accurate inference results when the sample size is moderate or even small. Second, missing values in individuals or time points are very common for the spatial panel data in practice. For example, in our real application, there are missing observations of AQI for certain cities (for example, Yantai), 
and we adopt the likewise deletion method for simplicity. However, the likewise deletion may result in information loss. Hence, it is beneficial to consider an estimation procedure in the presence of missing values at the response. Third, a location-scale model as given in (2.2) is specified in this paper, and a more general quantile regression model can be considered in the following form

$$
Q_{\theta_{i}}\left(\tau \mid x_{1 i}, \cdots, x_{p i}\right)=\tilde{\psi}_{0}(\tau)+\sum_{\ell=1}^{p} \tilde{\psi}_{\ell}(\tau) x_{\ell i},
$$

where $\tilde{\psi}_{\ell}(\cdot)$ 's are the quantile dependent coefficients. We leave these topics for future research.

\section{Supplementary Materials}

The supplementary material contains a bootstrapping procedure for QMLE, additional results for simulation, detailed forms of the matrices $\Sigma_{N}, \Sigma_{N}^{\star}, \Omega_{N T}^{-1}\left(\boldsymbol{\beta}^{\star}\right)$ and the vector $\boldsymbol{Y}_{-1}$, as well as technical details for Theorems 1- 6 and Propositions 1 - 2 with Lemmas 1 - 10.

\section{Acknowledgements}

We are deeply grateful to the co-editor, the associate editor, and two anonymous referees for their valuable comments and suggestions, which led to substantial improvement of this article. This research was partly supported by the National Natural Science Foundation of China grant (11971292, 11690012 and 12001355), Shanghai Pujiang Program 2019PJC051, Chenguang Program 19CG44, and Shanghai Research Center for Data Science and Decision Technology. The authors make equal contributions to this paper and their names are listed in an alphabetical order. 


\section{References}

Baltagi, B. H., P. Egger, and M. Pfaffermayr (2013). A generalized spatial panel data model with random effects. Econometric Reviews 32, 650-685.

Baltagi, B. H., B. C. Jung, and S. H. Song (2010). Testing for heteroskedasticity and serial correlation in a random effects panel data model. Journal of Econometrics 154, 122-124.

Bowatte, G., C. Lodge, A. J. Lowe, B. Erbas, J. Perret, M. J. Abramson, M. Matheson, and S. C. Dharmage (2015). The influence of childhood traffic-related air pollution exposure on asthma, allergy and sensitization: a systematic review and a meta-analysis of birth cohort studies. Allergy 70, 245-256.

Busch, P.-O. and H. Jörgens (2005). International patterns of environmental policy change and convergence. European Environment 15, 80-101.

Cakmak, S., R. Dales, J. Leech, and L. Liu (2011). The influence of air pollution on cardiovascular and pulmonary function and exercise capacity: Canadian health measures survey (CHMS). Environmental Research 111, 13091312 .

Canay, I. A. (2011). A simple approach to quantile regression for panel data. The Econometrics Journal 14, 368-386.

Chen, J., B. Wang, S. Huang, and M. Song (2020). The influence of increased population density in China on air pollution. Science of the Total Environment 735, 139456.

Chen, N. and L. Xu (2017). Relationship between air quality and economic development in the provincial capital cities of China. Environmental Science and Pollution Research 24, 2928-2935.

Chernozhukov, V. and C. Hansen (2006). Instrumental quantile regression inference for structural and treatment effect models. Journal of Econometrics 132, 491-525.

Cramer, J. C. (1998). Population growth and air quality in California. Demography 35, 45-56.

De Bruyn, S. M., J. C. van den Bergh, and J. B. Opschoor (1998). Economic growth and emissions: reconsidering 
the empirical basis of environmental Kuznets curves. Ecological Economics 25, 161-175.

Doob, J. L. (1935). The limiting distributions of certain statistics. The Annals of Mathematical Statistics 6, 160-169.

Giri, D., M. Krishna, and P. Adhikary (2008). The influence of meteorological conditions on PM10 concentrations in Kathmandu Valley. International Journal of Environmental Research 2, 49-60.

Gulia, S., S. S. Nagendra, M. Khare, and I. Khanna (2015). Urban air quality management-A review. Atmospheric Pollution Research 6, 286-304.

Hansen, B. (2021). Econometrics. University of Wisconsin. Available at https://www.ssc.wisc.edu/ bhansen/ econometrics/Econometrics.pdf.

Johnson, S. G. (2021). The nlopt nonlinear-optimization package. http://github.com/stevengj/nlopt.

Kapoor, M., H. H. Kelejian, and I. R. Prucha (2007). Panel data models with spatially correlated error components. Journal of Econometrics 140, 97-130.

Kelejian, H. H. and I. R. Prucha (1998). A generalized spatial two-stage least squares procedure for estimating a spatial autoregressive model with autoregressive disturbances. The Journal of Real Estate Finance and Economics 17, $99-121$.

Kelejian, H. H. and I. R. Prucha (1999). A generalized moments estimator for the autoregressive parameter in a spatial model. International Economic Review 40, 509-533.

Kelejian, H. H. and I. R. Prucha (2001). On the asymptotic distribution of the Moran I test statistic with applications. Journal of Econometrics 104, 219-257.

Kelejian, H. H. and I. R. Prucha (2010). Specification and estimation of spatial autoregressive models with autoregressive and heteroskedastic disturbances. Journal of Econometrics 157, 53-67.

Koenker, R. and G. J. Bassett (1978). Regression quantiles. Econometrica 46, 33-50.

Koenker, R. and Q. Zhao (1994). L-estimatton for linear heteroscedastic models. Journaltitle of Nonparametric 
Statistics 3, 223-235.

Lee, C. C., T. J. Ballinger, and N. A. Domino (2012). Utilizing map pattern classification and surface weather typing to relate climate to the Air Quality Index in Cleveland, Ohio. Atmospheric Environment 63, 50-59.

Lee, L.-F. (2004). Asymptotic distributions of quasi-maximum likelihood estimators for spatial autoregressive models. Econometrica 72, 1899-1925.

Lee, L.-F. and J. Yu (2010). Estimation of spatial autoregressive panel data models with fixed effects. Journal of Econometrics 154, 165-185.

Lee, L.-F. and J. Yu (2014). Efficient gmm estimation of spatial dynamic panel data models with fixed effects. Journal of Econometrics 180, 174-197.

Li, L., Y. Lei, S. Wu, C. He, J. Chen, and D. Yan (2018). Impacts of city size change and industrial structure change on $\mathrm{CO}_{2}$ emissions in Chinese cities. Journal of Cleaner Production 195, 831-838.

Li, L. and Z. Yang (2021). Spatial dynamic panel data models with correlated random effects. Journal of Econometrics 221, 424-454.

Mundlak, Y. (1978). On the pooling of time series and cross section data. Econometrica 46, 69-85.

Neyman, J. and E. L. Scott (1948). Consistent estimates based on partially consistent observations. Econometrica 16, 1-32.

Shi, W. and L.-F. Lee (2017). Spatial dynamic panel data models with interactive fixed effects. Journal of Econometrics $197,323-347$.

Stern, D. I., M. S. Common, and E. B. Barbier (1996). Economic growth and environmental degradation: the environmental Kuznets curve and sustainable development. World Development 24, 1151-1160.

Streck, C. (2004). New partnerships in global environmental policy: The clean development mechanism. The Journal of Environment \& Development 13, 295-322. 
Su, L. and Z. Yang (2015). QML estimation of dynamic panel data models with spatial errors. Journal of Econometrics 185, 230-258.

Wang, S. and J. Hao (2012). Air quality management in China: Issues, challenges, and options. Journal of Environmental Sciences 24, 2-13.

Yang, L., D. Wang, S. Cheng, Z. Wang, Y. Zhou, X. Zhou, and W. Wang (2007). Influence of meteorological conditions and particulate matter on visual range impairment in Jinan, China. Science of the Total Environment 383, 164173.

Yu, J., R. De Jong, and L.-F. Lee (2008). Quasi-maximum likelihood estimators for spatial dynamic panel data with fixed effects when both $n$ and $T$ are large. Journal of Econometrics 146, 118-134.

Yu, Y. and H. Liu (2020). Economic growth, industrial structure and nitrogen oxide emissions reduction and prediction in China. Atmospheric Pollution Research 11, 1042-1050.

Zhao, Z. and Z. Xiao (2014). Efficient regressions via optimally combining quantile information. Econometric Theory 30, 1272-1314.

Zhou, J., J. Liu, F. Wang, and H. Wang (2022). Autoregressive model with spatial dependence and missing data. Journal of Business \& Economic Statistics 40, 28-34.

Zhu, Q., Y. Zheng, and G. Li (2018). Linear double autoregression. Journal of Econometrics 20\%, $162-174$.

Xingdong Feng, Shanghai University of Finance and Economics, School of Statistics and Management

E-mail: feng.xingdong@mail.shufe.edu.cn

Wenyu Li, Shanghai University of Finance and Economics, School of Statistics and Management

E-mail: liwenyu2018@163.sufe.edu.cn

Qianqian Zhu, Shanghai University of Finance and Economics, School of Statistics and Management

E-mail: zhu.qianqian@mail.shufe.edu.cn 


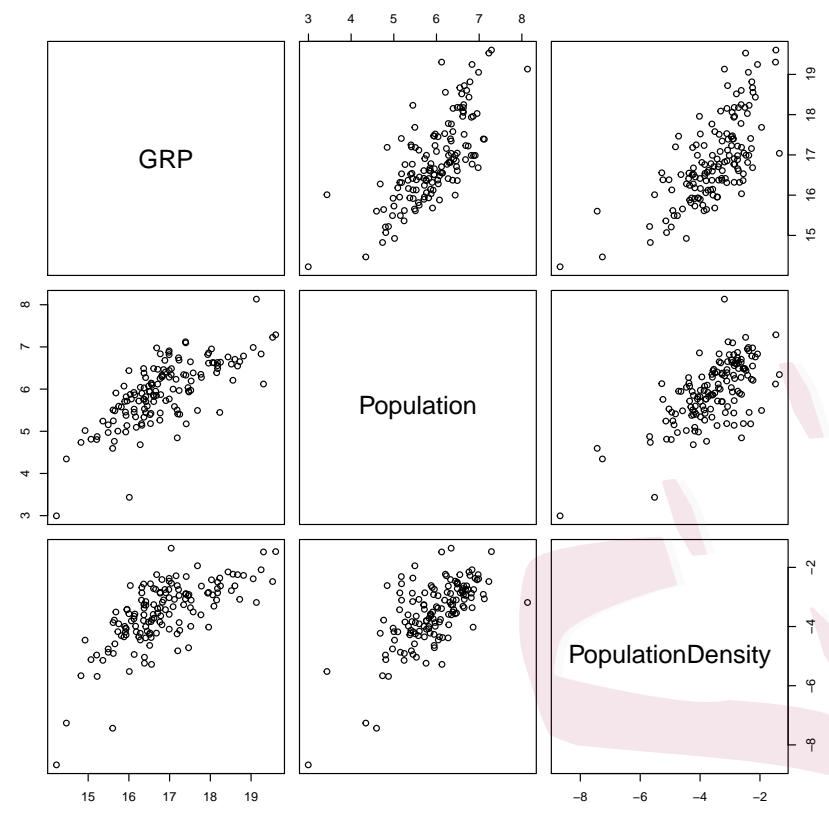

Figure 1: Scatterplots of log-transformed variables "GRP", "population" and "population density".
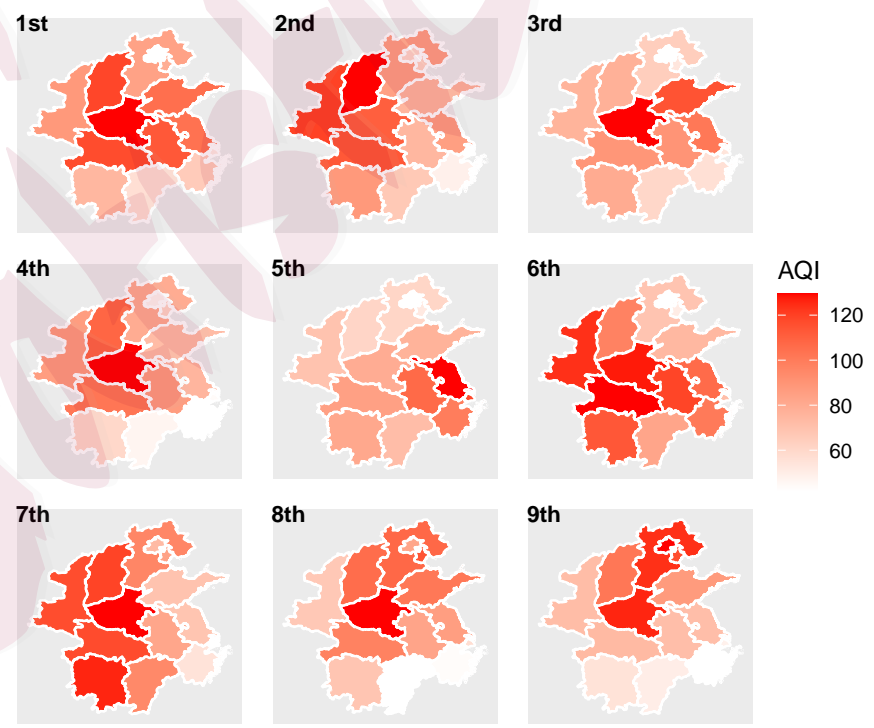

Figure 2: Heat maps of the AQI of a part of central and eastern China from the first to ninth weeks in 2018 . 
Table 1: Biases, ESDs and ASDs of the QMLE $\widehat{\zeta}$ when the innovations $\left\{\eta_{i}\right\}$ follow the standard normal or uniform distribution.

\begin{tabular}{|c|c|c|c|c|c|c|c|c|}
\hline & \multirow[b]{2}{*}{$N$} & \multirow[b]{2}{*}{$T$} & \multicolumn{3}{|c|}{$N(0,1)$} & \multicolumn{3}{|c|}{$U(-\sqrt{3}, \sqrt{3})$} \\
\hline & & & Bias & ESD & ASD & Bias & ESD & ASD \\
\hline \multirow[t]{3}{*}{$\alpha$} & 20 & 20 & 0.0021 & 0.0234 & 0.0398 & 0.0029 & 0.0232 & 0.0341 \\
\hline & 20 & 50 & 0.0007 & 0.0146 & 0.0400 & 0.0008 & 0.0141 & 0.0332 \\
\hline & 100 & 50 & 0.0006 & 0.0080 & 0.0089 & 0.0006 & 0.0079 & 0.0088 \\
\hline \multirow[t]{3}{*}{$\gamma_{1}$} & 20 & 20 & 0.0020 & 0.0511 & 0.0561 & 0.0013 & 0.0516 & 0.0571 \\
\hline & 20 & 50 & 0.0019 & 0.0336 & 0.0381 & 0.0026 & 0.0318 & 0.0371 \\
\hline & 100 & 50 & 0.0002 & 0.0145 & 0.0147 & 0.0005 & 0.0144 & 0.0147 \\
\hline \multirow[t]{3}{*}{$\psi_{0}$} & 20 & 20 & 0.0089 & 0.3737 & 1.2303 & 0.0091 & 0.3930 & 0.8101 \\
\hline & 20 & 50 & 0.0292 & 0.3561 & 1.8826 & 0.0201 & 0.3966 & 2.1397 \\
\hline & 100 & 50 & 0.0040 & 0.1537 & 0.1609 & 0.0045 & 0.1519 & 0.1596 \\
\hline \multirow[t]{3}{*}{$\psi_{1}$} & 20 & 20 & 0.0404 & 0.6824 & 1.2488 & 0.0161 & 0.6284 & 0.9061 \\
\hline & 20 & 50 & 0.0018 & 0.6537 & 4.9535 & 0.0149 & 0.6501 & 3.0061 \\
\hline & 100 & 50 & 0.0072 & 0.2782 & 0.2731 & 0.0100 & 0.2742 & 0.2735 \\
\hline \multirow[t]{3}{*}{$\sigma_{\varepsilon}^{2}$} & 20 & 20 & 0.0039 & 0.0714 & 0.1266 & 0.0086 & 0.0752 & 0.1138 \\
\hline & 20 & 50 & 0.0013 & 0.0464 & 0.1113 & 0.0027 & 0.0449 & 0.0913 \\
\hline & 100 & 50 & 0.0001 & 0.0201 & 0.0377 & 0.0003 & 0.0193 & 0.0366 \\
\hline \multirow[t]{3}{*}{$\lambda$} & 20 & 20 & 0.0030 & 0.0237 & 0.0435 & 0.0037 & 0.0235 & 0.0375 \\
\hline & 20 & 50 & 0.0008 & 0.0147 & 0.0428 & 0.0010 & 0.0141 & 0.0346 \\
\hline & 100 & 50 & 0.0006 & 0.0080 & 0.0089 & 0.0006 & 0.0079 & 0.0088 \\
\hline \multirow[t]{3}{*}{$\beta_{0}^{\star}$} & 20 & 20 & 0.1805 & 0.5059 & 2.7118 & 0.1295 & 0.4365 & 1.6301 \\
\hline & 20 & 50 & 0.1834 & 0.4967 & 7.5333 & 0.1041 & 0.4295 & 4.2356 \\
\hline & 100 & 50 & 0.0198 & 0.2087 & 0.2581 & 0.0177 & 0.1360 & 0.1718 \\
\hline \multirow[t]{3}{*}{$\beta_{1}^{\star}$} & 20 & 20 & 0.1256 & 0.9422 & 5.7388 & 0.0584 & 0.7995 & 3.0798 \\
\hline & 20 & 50 & 0.1419 & 0.9644 & 15.7608 & 0.0312 & 0.8020 & 9.5194 \\
\hline & 100 & 50 & 0.0015 & 0.4065 & 0.4525 & 0.0043 & 0.2630 & 0.2991 \\
\hline
\end{tabular}


Table 2: Biases, ESDs and ASDs of the WCQE $\widehat{\varphi}(\tau)$ at $\tau=0.25,0.5,0.75$, when the innovations $\left\{\eta_{i}\right\}$ follow the standard normal or uniform distribution.

\begin{tabular}{|c|c|c|c|c|c|c|c|c|c|}
\hline & & \multirow[b]{2}{*}{$N$} & \multirow[b]{2}{*}{$T$} & \multicolumn{3}{|c|}{$N(0,1)$} & \multicolumn{3}{|c|}{$U(-\sqrt{3}, \sqrt{3})$} \\
\hline & & & & Bias & ESD & ASD & Bias & ESD & ASD \\
\hline \multirow[t]{6}{*}{$\tau=0.25$} & $\varphi_{0}$ & 20 & 20 & 0.0303 & 0.4880 & 0.9059 & 0.1333 & 0.5239 & 0.9455 \\
\hline & & 100 & 50 & 0.0219 & 0.3287 & 0.3713 & 0.0479 & 0.3667 & 0.3934 \\
\hline & & 300 & 200 & 0.0081 & 0.1935 & 0.1970 & 0.0144 & 0.2116 & 0.2101 \\
\hline & $\varphi_{1}$ & 20 & 20 & 0.0913 & 0.7667 & 1.6977 & 0.0247 & 0.8428 & 1.7688 \\
\hline & & 100 & 50 & 0.0585 & 0.5937 & 0.7213 & 0.0496 & 0.6657 & 0.7625 \\
\hline & & 300 & 200 & 0.0225 & 0.3896 & 0.4091 & 0.0056 & 0.4270 & 0.4368 \\
\hline \multirow[t]{6}{*}{$\tau=0.5$} & $\varphi_{0}$ & 20 & 20 & 0.0164 & 0.2520 & 0.8681 & 0.0037 & 0.2654 & 1.0016 \\
\hline & & 100 & 50 & 0.0038 & 0.1092 & 0.3529 & 0.0035 & 0.1334 & 0.4541 \\
\hline & & 300 & 200 & 0.0045 & 0.0589 & 0.1851 & 0.0041 & 0.0750 & 0.2427 \\
\hline & $\varphi_{1}$ & 20 & 20 & 0.0143 & 0.3411 & 1.6275 & 0.0253 & 0.4375 & 1.8757 \\
\hline & & 100 & 50 & 0.0104 & 0.1718 & 0.6852 & 0.0028 & 0.2384 & 0.8802 \\
\hline & & 300 & 200 & 0.0052 & 0.1125 & 0.3844 & 0.0015 & 0.1558 & 0.5042 \\
\hline \multirow[t]{6}{*}{$\tau=0.75$} & $\varphi_{0}$ & 20 & 20 & 0.0336 & 0.4843 & 0.8838 & 0.1093 & 0.5027 & 0.9572 \\
\hline & & 100 & 50 & 0.0141 & 0.3197 & 0.3737 & 0.0511 & 0.3666 & 0.3981 \\
\hline & & 300 & 200 & 0.0082 & 0.1913 & 0.1960 & 0.0063 & 0.2080 & 0.2101 \\
\hline & $\varphi_{1}$ & 20 & 20 & 0.0864 & 0.7897 & 1.6561 & 0.0118 & 0.8468 & 1.7927 \\
\hline & & 100 & 50 & 0.0300 & 0.5683 & 0.7254 & 0.0517 & 0.6734 & 0.7715 \\
\hline & & 300 & 200 & 0.0126 & 0.3703 & 0.4069 & 0.0119 & 0.4215 & 0.4368 \\
\hline
\end{tabular}


Table 3: Biases, ESDs and ASDs of the three estimators of $\boldsymbol{\beta}_{0}$, i.e. $\check{\boldsymbol{\beta}}$ by the QMLE, the initial estimator $\widetilde{\boldsymbol{\beta}}_{c}$ and the WQAE $\widehat{\boldsymbol{\beta}}\left(\widehat{\boldsymbol{\pi}}_{o p t, K}\right)$, when the innovations $\left\{\eta_{i}\right\}$ follow the standard normal or uniform distribution.

\begin{tabular}{|c|c|c|c|c|c|c|c|c|}
\hline & \multirow[b]{2}{*}{$N$} & \multirow[b]{2}{*}{$T$} & \multicolumn{3}{|c|}{$N(0,1)$} & \multicolumn{3}{|c|}{$U(-\sqrt{3}, \sqrt{3})$} \\
\hline & & & Bias & ESD & ASD & Bias & ESD & ASD \\
\hline \multirow[t]{4}{*}{$\check{\beta}_{1}$} & 20 & 20 & 276805 & 827496 & $5 e+12$ & 146686 & 609974 & $3 e+12$ \\
\hline & 50 & 20 & 3181 & 100581 & $4 e+9$ & 3281 & 103752 & $4 e+9$ \\
\hline & 100 & 50 & 0.1638 & 0.7819 & 0.8772 & 0.0779 & 0.4386 & 0.5052 \\
\hline & 300 & 200 & 0.0340 & 0.3032 & 0.3548 & 0.0167 & 0.1942 & 0.2278 \\
\hline \multirow[t]{3}{*}{$\widetilde{\beta}_{1 c}$} & 20 & 20 & 0.5346 & 1.2984 & - & 0.4840 & 1.2580 & - \\
\hline & 100 & 50 & 0.2714 & 0.7978 & - & 0.2020 & 0.5663 & - \\
\hline & 300 & 200 & 0.0668 & 0.3621 & - & 0.0637 & 0.3044 & - \\
\hline \multirow[t]{3}{*}{$\widehat{\beta}_{1}$} & 20 & 20 & 0.5116 & 1.2505 & 1.1530 & 0.3418 & 1.1946 & 1.0260 \\
\hline & 100 & 50 & 0.1165 & 0.8976 & 0.4603 & 0.0669 & 0.5035 & 0.3477 \\
\hline & 300 & 200 & 0.0348 & 0.3578 & 0.2455 & 0.0606 & 0.2372 & 0.1730 \\
\hline
\end{tabular}

Table 4: Summary statistics for AQI, TEM $\left(0.1^{\circ} \mathrm{C}\right)$, PRE $(0.1 \mathrm{~mm})$, WIN $(0.1 \mathrm{~m} / \mathrm{s})$, GRP (10000 yuan) and Industry (\%).

\begin{tabular}{rrrrrrr}
\hline \hline & AQI & TEM & PRE & WIN & GRP & Industry \\
\hline Min & 13.264 & -283.000 & 0.000 & 4.714 & 1500100 & 18.270 \\
$\operatorname{Max}$ & 312.845 & 325.714 & 5113.000 & 78.286 & 326798700 & 72.900 \\
\hline
\end{tabular}


Table 5: Summary information of fitted coefficients for models (5.1) and (5.2).

\begin{tabular}{|c|c|c|c|c|}
\hline & Estimate & Std. Error & z statistic & p-value \\
\hline$\alpha$ & 0.178 & 0.027 & 6.593 & 0.000 \\
\hline$\lambda$ & 0.648 & 0.022 & 29.455 & 0.000 \\
\hline$\gamma_{1}$ & -0.096 & 0.015 & -6.400 & 0.000 \\
\hline$\gamma_{2}$ & -0.065 & 0.006 & -10.833 & 0.000 \\
\hline$\gamma_{3}$ & -0.078 & 0.013 & -6.000 & 0.000 \\
\hline$\psi_{0}$ & 0.171 & 0.194 & 0.881 & 0.378 \\
\hline$\psi_{1}$ & 0.078 & 0.088 & 0.886 & 0.375 \\
\hline$\psi_{2}$ & 0.063 & 0.036 & 1.750 & 0.080 \\
\hline$\varphi_{0}(0.25)$ & -0.066 & 0.103 & -0.641 & 0.522 \\
\hline$\varphi_{1}(0.25)$ & -0.016 & 0.028 & -0.571 & 0.568 \\
\hline$\varphi_{2}(0.25)$ & -0.046 & 0.024 & -1.917 & 0.055 \\
\hline$\varphi_{0}(0.75)$ & 0.030 & 0.216 & 0.139 & 0.890 \\
\hline$\varphi_{1}(0.75)$ & 0.021 & 0.058 & 0.362 & 0.717 \\
\hline$\varphi_{2}(0.75)$ & 0.014 & 0.049 & 0.286 & 0.775 \\
\hline
\end{tabular}

